\title{
CONTENTS OF VOLUME 82, 2018
}

\section{Message From the Editor-in-Chief}

1. Message From the Editor-in-Chief

Toyoaki Murohara

2. Editorial Statistics and Best Reviewers Award for 2017

Toyoaki Murohara

3. Circulation Journal Awards for the Year 2017

Toyoaki Murohara

4. Rapid Publication and Announcement of the 2018 JCS Meeting Activities

Toyoaki Murohara

5. Announcement of the 2018 JCS Meeting Activities (2)

Toyoaki Murohara

6. Message From the Editor-in-Chief

Toyoaki Murohara

7. Message From the Editor-in-Chief Toyoaki Murohara

8. Message From the Editor-in-Chief Toyoaki Murohara

\section{Reviews}

1. Strategy and Outcome of Catheter Ablation for Persistent Atrial Fibrillation - Impact of Progress in the Mapping and Ablation Technologies -

2. Metabolomic Analysis in Heart Failure

Ryutaro Ikegami, Ippei Shimizu, Yohko Yoshida, Tohru Minamino

3. Optical Coherence Tomography Guidance in Management of Acute Coronary Syndrome Caused by Plaque Erosion Haibo Jia, Takashi Kubo, Takashi Akasaka, Bo Yu

4. Type 2 Myocardial Infarction - An Evolving Entity -

Cian P. McCarthy, James L. Januzzi Jr, Hanna K. Gaggin

5. Registry Assessment of Peripheral Interventional Devices (RAPID) - Registry Assessment of Peripheral Interventional Devices Core Data Elements -

W. Schuyler Jones, Mitchell W. Krucoff, Pablo Morales, Rebecca W. Wilgus, Anne H. Heath, Mary F. Williams, James E. Tcheng, J. Danica Marinac-Dabic, Misti L. Malone, Terrie L. Reed, Rie Fukaya, Robert Lookstein, Nobuhiro Handa, Herbert D. Aronow, Daniel J. Bertges, Michael R. Jaff, Thomas T. Tsai, Joshua A. Smale, Margo J. Zaugg, Robert J. Thatcher, Jack L. Cronenwett, Durham NC, Silver Spring Md, Tokyo Japan, New York NY, Providence RI, Burlington Vt, Newton Mass, Denver Colo, Tempe Ariz, Santa Clara Calif, Minneapolis Minn, Lebanon NH

6. High-Frequency Echocardiography - Transformative Clinical and Research Applications in Humans, Mice, and Zebrafish -

Louis W. Wang, Scott Kesteven, Inken G. Huttner, Michael P. Feneley, Diane Fatkin

7. Inflammatory Mechanisms of Cardiovascular Remodeling Toshihisa Anzai

8. Rapid Globalization of Medical Device Clinical Development Programs in Japan - The Case of Drug-Eluting Stents Madoka Murakami, Yuka Suzuki, Toshiyoshi Tominaga

9. Best Treatment Strategies With Statins to Maximize the Cardiometabolic Benefits

Kyoung Im Cho, Ichiro Sakuma, Il Suk Sohn, Toshio Hayashi, Kazunori Shimada, Kwang Kon Koh

10. After the Dawn - Balloon Pulmonary Angioplasty for Patients With Chronic Thromboembolic Pulmonary Hypertension Aiko Ogawa, Hiromi Matsubara

11. Long Non-Coding RNAs as Key Regulators of Cardiovascular Diseases Koh Ono, Yasuhide Kuwabara, Takahiro Horie, Takeshi Kimura

12. Aging Modulates the Substrate and Triggers Remodeling in Atrial Fibrillation Yung-Kuo Lin, Yi-Ann Chen, Ting-I Lee, Yao-Chang Chen, Shih-Ann Chen, Yi-Jen Chen 
13. Clinical Usefulness of Wearable Cardioverter Defibrillator (WCD) and Current Understanding of Its Clinical Indication in Japan

Shinichi Niwano, Yukio Sekiguchi, Yosuke Ishii, Yuki Iwasaki, Ritsushi Kato, Hideo Okamura, Masahiko Takagi, Takashi Kurita, Takashi Nitta

14. First Approval of Improved Medical Device Conditional on Use-Result Survey in Japan - Regulatory Review of Polymer-Free Drug-Coated BioFreedom Coronary Stent -

Akihide Konishi, Mami Ho, Yuko Shirai, Haruki Shirato

15. Atherosclerotic Coronary Plaque Development Visualized by In Vivo Coronary Imaging Kenji Sakamoto, Suguru Nagamatsu, Eiichiro Yamamoto, Koichi Kaikita, Kenichi Tsujita

Subclinical Leaflet Thrombosis in Transcatheter Aortic Valve Replacement Detected by Multidetector Computed Tomography - A Review of Current Evidence -

Hashrul N. Rashid, Adam J. Brown, Liam M. McCormick, Ameera S. Amiruddin, Kim K. Be, James D. Cameron, Arthur Nasis, Robert P. Gooley

17. Contemporary Septal Reduction Therapy in Drug-Refractory Hypertrophic Obstructive Cardiomyopathy Yuichiro Maekawa, Keitaro Akita, Shuichiro Takanashi

8. Mesenchymal Stem/Stromal Cell-Based Therapy for Heart Failure - What Is the Best Source? Kazuya Kobayashi, Ken Suzuki

19. Design Strategies for Global Clinical Trials of Endovascular Devices for Critical Limb Ischemia (CLI) - A Joint USA-Japanese Perspective -

Hiroyoshi Yokoi, Mami Ho, Shin Iwamoto, Yuka Suzuki, Gary M. Ansel, Nobuyoshi Azuma, Nobuhiro Handa, Osamu Iida, Koji Ikeda, Fumiaki Ikeno, Norihiko Ohura, Kenneth Rosenfield, John Rundback, Hiroto Terashi, Takahiro Uchida, Yoshiaki Yokoi, Masato Nakamura, Michael R. Jaff

20. Cutting Edge of Brain Natriuretic Peptide (BNP) Research - The Diversity of BNP Immunoreactivity and Its Clinical Relevance -

Koichiro Kuwahara, Yasuaki Nakagawa, Toshio Nishikimi

21. Research Toolbox for Peripheral Arterial Disease - Minimally Invasive Assessment of the Vasculature and Skeletal Muscle -

Lee Stoner, Erik D. Hanson, Martin Gram, Jason D. Allen, Steven K. Malin

22. Emerging Role of Plasma Membranes in Vascular Endothelial Mechanosensing Kimiko Yamamoto, Joji Ando

23. Therapeutic Strategy for Gastrointestinal Bleeding in Patients With Left Ventricular Assist Device Teruhiko Imamura, Koichiro Kinugawa, Nir Uriel

\section{Congress Reports}

\section{AHA Report}

1. Report of the American Heart Association (AHA) Scientific Session 2017, Anaheim, California Yoshihiro Seo

\section{ACC Report}

2. Report of the Annual Scientific Session of the American College of Cardiology (ACC) 2018, Orlando Takuya Hashimoto, Junya Ako

\section{JCS Report}

3. Overview of the $82^{\text {nd }}$ Annual Scientific Meeting of the Japanese Circulation Society - Futurability: Pioneering the Future of Circulatory Medicine Hiroki Hata, Toru Kuratani, Takashi Shibuya, Koichi Toda, Takayoshi Ueno, Shigeru Miyagawa, Yasushi Yoshikawa, Kazuo Shimamura, Keiwa Kin, Daisuke Yoshioka, Masaki Taira, Koichi Maeda, Keitaro Domae, Yoshiki Sawa

\section{Editorials}

1. Who Will Achieve Stable Anticoagulation Therapy With Warfarin?

Yoshimori An, Masaharu Akao

2. Impact of Stent Type on the Long-Term Prognosis of Patients With Vasospastic Angina - Can We Judge a Stent Just by Its Cover? -

Hiroto Suzuyama, Tomohiro Sakamoto, Koich Nakao

3. Has the Development of Drug-Eluting Stents Ended With Limus-Eluting Stents? Ken Kozuma

4. Unsolved Antiatherogenic Mechanism of n-3 Polyunsaturated Fatty Acids Masa-aki Kawashiri, Hayato Tada, Masakazu Yamagishi

5. Novel Therapeutic Target of Anticoagulation Therapy in Patients With Atrial Fibrillation - Cognitive Decline and Dementia -

Eitaro Kodani, Takehiko Nagao 
6. Impact of Anemia on Left Ventricular Reverse Remodeling in Response to Carvedilol Akiomi Yoshihisa

7. Left Ventricular Fiber Structure and Myocardial Deformation for Assessment of Cardiotoxicity Ikuo Hashimoto, Fukiko Ichida

8. Risk of Cardiovascular Disease After Earthquake Disaster Seiji Hokimoto

9. Progression of Functional Tricuspid Regurgitation With Aortic Valve Stenosis After Intervention Wataru Tatsuishi, Kiyoharu Nakano

10. Current Status of Rotational Atherectomy in the Drug-Eluting Stent Era Tadayuki Uetani, Tetsuya Amano

11. Obstacles to Optimal Lipid-Lowering Therapy — Any Solution? Yu Kataoka

12. Exploring Deep Into the Coronary Microcirculation of Patients With Microvascular Angina Masami Goto

13. Towards Effective Cost-Effectiveness Analysis in Atherosclerotic Cardiovascular Disease Prevention Kouji Kajinami

14. Anticoagulation Therapy After Left Ventricular Assist Device Implantation Yoshihisa Tanoue, Takeo Fujino, Akira Shiose

15. Cutting Edge Science and Medicine of Adenosine in Patients With Heart Failure Hiroshi Asanuma, Masafumi Kitakaze

16. Is There Any Impact of ST Re-elevation on Patients With Acute Myocardial Infarction Following Reperfusion Therapy? Kengo Tanabe

17. Should Measures of High-Density Lipoprotein Function Be Added to the Low-Density Lipoprotein Cholesterol Target Level-Based Guidelines for Prevention of Atherosclerotic Disease? Ichiro Sakuma, Katsunori Ikewaki

18. Towards the Application of Endovascular Treatment for Superficial Femoral Artery Atsubumi Murakami

19. Role of Tissue Factor in Vascular Failure

Teruo Inoue, Masashi Sakuma, Shigeru Toyoda, Koichi Node

20. Detrimental Effects of Insufficient Warfarin Therapy - A Warning Against Imprudent Use of Warfarin for Atrial Fibrillation Patients -

Junya Kuroda

21. Mesoangioblasts - A Newcomer in Cell-Based Treatment Strategy for Cardiovascular Disease? Ken-ichiro Sasaki, Yoshihiro Fukumoto

22. Are All New-Generation Drug-Eluting Stents Equal? - Long-Term Follow-up Is Necessary Kengo Tanabe

23. Association Between Glycemic Control and Incident Stroke - Overview From an Epidemiologic Study Takumi Hirata, Tomonori Okamura

24. Nutritional Status Assessment as a Potential Prognostic Indicator in Patients Before the Development of Symptomatic Heart Failure Tomohisa Nagoshi, Michihiro Yoshimura

25. Hepatitis C Virus and Cardiovascular Disease Kenji Onoue, Yoshihiko Saito

26. Appropriate Use of Urinary Catheter in Acute Heart Failure Patients Tatsuo Aoki

27. Gut Microbial Dysbiosis in Heart Failure - Is It a Future Therapeutic Target or Not? Tomoya Yamashita, Tomohiro Hayashi, Naofumi Yoshida, Ken-ichi Hirata

28. Smoking and Incident Hypertension - Importance of Cotinine-Verified Smoking Status Toshiaki Ebina

29. What Causes Pulmonary Arterial Hypertension in Down Syndrome With Congenital Heart Disease? Susumu Hosokawa, Rebecca R. Vanderpool, Taku Ishii, Mitsunori Nishiyama, Shozaburo Doi

30. Improving Progenitor Cell Selection to Promote Therapeutic Angiogenesis in Patients With Critical Limb Ischemia Alain Rivard

31. Is Female Sex Always a Risk for Bleeding? Hidehira Fukaya, Junya Ako

32. Prevention of Periprocedural Myocardial Injury During Percutaneous Coronary Intervention in Patients With Stable Coronary Artery Disease Shin Ito, Masafumi Kitakaze

33. Smoke-Free Legislation and Acute Coronary Syndrome Hiroshi Yamato, Ying Jiang 
34. Are Sodium-Glucose Cotransporter 2 Inhibitors Really Useful and Safe for Advanced Heart Failure?

Takahiro Okumura, Toyoaki Murohara

35. Monitoring Changes in Ejection Fraction in Patients With Heart Failure and Mid-Range Ejection Fraction Masahiko Kato, Kazuhiro Yamamoto

36. The Vicious Combination of Left Ventricular Diastolic Dysfunction and Frailty Tomoko Ishizu

37. Limited Extent of Pleiotropic Effects Mediated by Dipeptidyl Peptidase-4 Inhibitors in Patients With Diabetes Mellitus Kensuke Noma, Yasuki Kihara, Yukihito Higashi

38. Frailty Assessment - An Indispensable Component of Transcatheter Aortic Valve Implantation Kentaro Hayashida

39. Risk Factors for New-Onset Atrial Fibrillation in the General Population and Patients Who Visit Hospital Eitaro Kodani

40. Sex Difference in Clinical Recurrence After Catheter Ablation in Young Patients With Atrial Fibrillation - What Is the Underlying Mechanism? Hiroya Mizuno

41. They Are Not Monozygotic Twins - Long QT Syndrome Type 1 (LQT1) and Catecholaminergic Polymorphic Ventricular Tachycardia (CPVT) Hiroshi Morita

42. Pretreatment of Endothelial Progenitor Cells for Effective Cell Therapy Ken-ichiro Sasaki, Yoshihiro Fukumoto

43. A Lesson From the Thalidomide Tragedy - The Past Is Never Dead. It's Not Even Past. William Faulkner, From "Requiem for a Nun"-

Kenta Yashiro, Shigeru Miyagawa, Yoshiki Sawa

44. Bicuspid Aortic Valve-Associated Aortic Dilatation - What Is the Mechanism of Bicuspid Aortopathy? Tomoya Yamashita, Tomohiro Hayashi, Tokiko Tabata, Ken-ichi Hirata

45. Fixation or Disinfection? Takashi Kunihara

46. Epicardial Fat and Pericardial Fat Surrounding the Heart Have Different Characteristics Yukina Hirata, Hirotsugu Yamada, Masataka Sata

47. Early Prediction of Cardiotoxicity From Cancer Chemotherapy - Can Baseline Strain Identify High-Risk Patients? Koki Nakanishi, Masao Daimon

$$
\text { . }
$$

48. PARALLEL-HF - A Paradigm Shift for Heart Failure Treatment in Japan? Kyung-Duk Min, Masafumi Kitakaze

49. Trade-Off Between Lipid-Lowering Therapy and Costs in Patients With Cardiovascular Disease Hideki Ishii, Toyoaki Murohara

50. More Benefits From Endovascular Thrombectomy in Patients With Atrial Fibrillation? Masatoshi Koga

51. Phenotype and Prognosis of the Lamin A/C Gene ( $L M N A)$ Mutation Carriers in Japan Noboru Fujino, Kenshi Hayashi, Kenji Sakata, Akihiro Nomura, Masa-aki Kawashiri

52. Is Transesophageal Echocardiography Necessary in Every Case of Atrial Fibrillation Ablation? Hideharu Okamatsu, Ken Okumura

53. Importance of Risk Control on the Incidence of Aortic Artery Disease-Related Disease in Epidemiological Studies Hisashi Adachi

54. Alternative Exercise Intervention for Patients With Severe Peripheral Artery Disease - Akt1-Induced Blood Flow Recovery Kentaro Jujo, Nobuhisa Hagiwara

55. Dementia and Cardiovascular Surgery Akira Sezai

56. Tobacco and Non-Communicable Diseases Control in Japan Tetsutaro Matayoshi, Takahiro Tabuchi, Iwao Gohma, Takahiro Shimizu

57. Cardiac Allograft Vasculopathy - Heart Transplantation Provides Insights Into Pathogenesis and Treatment of Arteriosclerosis Shinichi Nunoda

\section{Late Breaking Clinical Trial (JCS 2018)}

1. Tailored Adjunctive Cilostazol Therapy Based on CYP2C19 Genotyping in Patients With Acute Myocardial Infarction - The CALDERA-GENE Study -

Koichi Kaikita, Hiromi Yoshimura, Masanobu Ishii, Takashi Kudoh, Yoshihiro Yamada, Eiichiro Yamamoto, Yasuhiro Izumiya, Sunao Kojima, Hideki Shimomura, Ryusuke Tsunoda, Kunihiko Matsui, Hisao Ogawa, Kenichi Tsujita for the CALDERA-GENE Investigators 
2. Application of a Fresh Decellularized Pulmonary Allograft for Pulmonary Valve Replacement in Japan Hideto Ozawa, Takayoshi Ueno, Masaki Taira, Koichi Toda, Toru Kuratani, Yoshiki Sawa

3. Percutaneous WATCHMAN Left Atrial Appendage Closure for Japanese Patients With Nonvalvular Atrial Fibrillation at Increased Risk of Thromboembolism - First Results From the SALUTE Trial -

Kazutaka Aonuma, Hiro Yamasaki, Masato Nakamura, Tatsushi Ootomo, Morimasa Takayama, Kenji Ando, Kenzo Hirao, Yoshihiro Morino, Kentaro Hayashida, Kengo Kusano, Michael L. Main, Shigeru Saito

Late Breaking Cohort Study (JCS 2018)

1. Anticoagulation Therapy for Venous Thromboembolism in the Real World - From the COMMAND VTE Registry -

Yugo Yamashita, Takeshi Morimoto, Hidewo Amano, Toru Takase, Seiichi Hiramori, Kitae Kim, Takashi Konishi, Masaharu Akao, Yohei Kobayashi, Takeshi Inoue, Maki Oi, Toshiaki Izumi, Kotaro Takahashi, Tomohisa Tada, Po-Min Chen, Koichiro Murata, Yoshiaki Tsuyuki, Hiroshi Sakai, Syunsuke Saga, Tomoki Sasa, Jiro Sakamoto, Chinatsu Yamada, Minako Kinoshita, Kiyonori Togi, Tomoyuki Ikeda, Katsuhisa Ishii, Kazuhisa Kaneda, Hiroshi Mabuchi, Hideo Otani, Kensuke Takabayashi, Mamoru Takahashi, Hiroki Shiomi, Takeru Makiyama, Koh Ono, Takeshi Kimura on behalf of COMMAND VTE Registry Investigators

2. Clinical Characteristics, Management, and Outcomes of Japanese Patients Hospitalized for Heart Failure With Preserved Ejection Fraction - A Report From the Japanese Heart Failure Syndrome With Preserved Ejection Fraction (JASPER) Registry -

Toshiyuki Nagai, Tsutomu Yoshikawa, Yoshihiko Saito, Yasuchika Takeishi, Kazuhiro Yamamoto, Hisao Ogawa, Toshihisa Anzai on behalf of the JASPER Investigators

\section{Original Articles}

Aortic Disease (6)

1. Midkine Promotes Atherosclerotic Plaque Formation Through Its Pro-Inflammatory, Angiogenic and Anti-Apoptotic Functions in Apolipoprotein E-Knockout Mice

Yoshio Takemoto, Mitsuru Horiba, Masahide Harada, Kazuma Sakamoto, Kyosuke Takeshita, Toyoaki Murohara, Kenji Kadomatsu, Kaichiro Kamiya

2. MicroRNA-182 Promotes Lipoprotein Lipase Expression and Atherogenesisby Targeting Histone Deacetylase 9 in Apolipoprotein E-Knockout Mice

Hai-Peng Cheng, Duo Gong, Zhen-Wang Zhao, Ping-Ping He, Xiao-Hua Yu, Qiong Ye, Chong Huang, Xin Zhang, Ling-Yan Chen, Wei Xie, Min Zhang, Liang Li, Xiao-Dan Xia, Xin-Ping Ouyang, Yu-Lin Tan, Zong-bao Wang, Guo-Ping Tian, Xi-Long Zheng, Wei-Dong Yin, Chao-Ke Tang

3. Preliminary Evaluation of Aortic Aneurysm Screening Using Oscillometric Device Equipped With Novel Algorithm Analyzing Pulse Wave Transfer

Shigeo Ichihashi, Tomoko Hashimoto, Shinichi Iwakoshi, Kenji Obayashi, Keigo Saeki, Kimihiko Kichikawa

4. Aneurysm Wall Enhancement Detected by Contrast Computed Tomography Scan Is Associated With Aneurysm Shrinkage After Endovascular Aneurysm Repair for Abdominal Aortic Aneurysm

Eisaku Ito, Naoki Toya, Soichiro Fukushima, Yuri Murakami, Tadashi Akiba, Takao Ohki

5. Activation of the AKT Pathway in the Ascending Aorta With Bicuspid Aortic Valve

Yuichiro Hirata, Hiroki Aoki, Takahiro Shojima, Kazuyoshi Takagi, Tohru Takaseya, Kohji Akasu, Satoru Tobinaga, Yoshihiro Fukumoto, Hiroyuki Tanaka

6. Positive Association of High Leptin Level and Abdominal Aortic Calcification in Men - The Prospective MINOS Study -

Pawel Szulc, Ez Zoubir Amri, Annie Varennes, Patricia Panaia-Ferrari, Eric Fontas, Joëlle Goudable, Roland Chapurlat, Véronique Breuil

Arrhythmia/Electrophysiology (42)

7. Predictors of International Normalized Ratio Variability in Patients With Atrial Fibrillation Under Warfarin Therapy Yoshimi Numao, Shinya Suzuki, Takuto Arita, Naoharu Yagi, Takayuki Otsuka, Koichi Sagara, Hiroaki Semba, Kenichi Sasaki, Hiroto Kano, Shunsuke Matsuno, Yuko Kato, Tokuhisa Uejima, Yuji Oikawa, Kazuyuki Nagashima, Hajime Kirigaya, Takashi Kunihara, Junji Yajima, Tadanori Aizawa, Takeshi Yamashita

8. Identification of Left Atrial Appendage Thrombi in Patients With Persistent and Long-Standing Persistent Atrial Fibrillation Using Intra-Cardiac Echocardiography and Cardiac Computed Tomography

Yukinori Ikegami, Kojiro Tanimoto, Kohei Inagawa, Yasuyuki Shiraishi, Jun Fuse, Munehisa Sakamoto, Yukihiko Momiyama, Seiji Takatsuki

9. Prolonged Right Ventricular Ejection Delay in Brugada Syndrome Depends on the Type of SCN5A Variant

- Electromechanical Coupling Through Tissue Velocity Imaging as a Bridge Between Genotyping and Phenotyping Sophie C.H. Van Malderen, Dorien Daneels, Dirk Kerkhove, Uschi Peeters, Dominic A.M.J. Theuns, Steven Droogmans, Guy Van Camp, Caroline Weytjens, Martine Biervliet, Maryse Bonduelle, Sonia Van Dooren, Pedro Brugada 
10. Arrhythmogenic Delayed Afterdepolarizations Are Promoted by Severe Hypothermia But Not Therapeutic Hypothermia

Hidehira Fukaya, Joseph S. Piktel, Xiaoping Wan, Bradley N. Plummer, Kenneth R. Laurita, Lance D. Wilson

11. Association of the Clinical and Genetic Factors With Superior Vena Cava Arrhythmogenicity in Atrial Fibrillation Yusuke Ebana, Junichi Nitta, Yoshihide Takahashi, Shinsuke Miyazaki, Masahito Suzuki, Lian Liu, Kenzo Hirao, Eiichiro Kanda, Mitsuaki Isobe, Tetsushi Furukawa

12. Isolated Late Activation Detected by Magnetocardiography Predicts Future Lethal Ventricular Arrhythmic Events in Patients With Arrhythmogenic Right Ventricular Cardiomyopathy

Yoshitaka Kimura, Hiroshi Takaki, Yuko Y. Inoue, Yasutaka Oguchi, Tomomi Nagayama, Takahiro Nakashima, Shoji Kawakami, Satoshi Nagase, Takashi Noda, Takeshi Aiba, Wataru Shimizu, Shiro Kamakura, Masaru Sugimachi, Satoshi Yasuda, Hiroaki Shimokawa, Kengo Kusano

13. Effects and Risks of Heparin Bridging and Different Modes of Interruption/Resumption of Rivaroxaban on Perioperative Complications of Catheter Ablation for Atrial Fibrillation - Another Analysis of the JACRE-R Registry -

Kenzo Hirao, Kazutaka Aonuma, Koichiro Kumagai, Koichi Inoue, Masaomi Kimura, Yasushi Miyauchi, Eiki Tsushima, Ken Okumura for the JACRE Investigators

14. Outcomes of Rate-Control Treatment in Patients With Atrial Fibrillation and Heart Failure - A Nationwide Cohort Study -

Hee Tae Yu, Pil-Sung Yang, Hancheol Lee, Seng Chan You, Tae-Hoon Kim, Jae-Sun Uhm, Jong-Youn Kim, Hui-Nam Pak, Moon-Hyoung Lee, Boyoung Joung

15. Predictors of a Persistent Status of Pulmonary Vein Electrical Isolation by a Cryoballoon Application for DrugRefractory Atrial Fibrillation

Naohiko Kawaguchi, Kaoru Okishige, Yasuteru Yamauchi, Manabu Kurabayashi, Kenzo Hirao

16. Does Size Matter? Cryoballoon-Based Pulmonary Vein Isolation Using a Novel 25-mm Circular Mapping Catheter Bruno Reissmann, Michael Schlüter, Francesco Santoro, Tilman Maurer, Christian-Hendrik Heeger, Christine Lemes, Thomas Fink, Johannes Riedl, Andreas Rillig, Shibu Mathew, Feifan Ouyang, Karl-Heinz Kuck, Andreas Metzner

17. Silent Cerebral Infarction in East Asian vs. Non-Asian Atrial Fibrillation Patients - Meta-Analysis Keitaro Senoo, Yusuke Kondo, Yoshio Kobayashi, Gregory YH Lip

18. Efficacy and Safety of Figure-of-Eight Suture for Hemostasis After Radiofrequency Catheter Ablation for Atrial Fibrillation

Masato Okada, Koichi Inoue, Koji Tanaka, Yuichi Ninomiya, Yuko Hirao, Takafumi Oka, Nobuaki Tanaka, Hiroyuki Inoue, Ryo Nakamaru, Yasushi Koyama, Atsunori Okamura, Katsuomi Iwakura, Yasushi Sakata, Kenshi Fujii

19. Combined Analysis of Human and Experimental Murine Samples Identified Novel Circulating MicroRNAs as Biomarkers for Atrial Fibrillation

Yu Natsume, Kasumi Oaku, Kentaro Takahashi, Wakana Nakamura, Ai Oono, Satomi Hamada, Masahiro Yamazoe, Kensuke Ihara, Takeshi Sasaki, Masahiko Goya, Kenzo Hirao, Tetsushi Furukawa, Tetsuo Sasano

20. Contiguity Between Ablation Lesions and Strict Catheter Stability Settings Assessed by VISITAG ${ }^{\text {TM }}$ Module Improve Clinical Outcomes of Paroxysmal Atrial Fibrillation Ablation - Results From the VISITALY Study -

Giulio Zucchelli, Giusy Sirico, Luca Rebellato, Massimiliano Marini, Giuseppe Stabile, Maurizio Del Greco, Antonello Castro, Ermenegildo De Ruvo, Ezio Soldati, Gianluca Zingarini, Salvatore Ocello, Elisabetta Daleffe, Massimo Mantica, Claudio Pandozi, Massimiliano Maines, Fabrizio Guarracini, Maria Grazia Bongiorni

21. Impact of B-Type Natriuretic Peptide Level on Risk Stratification of Thromboembolism and Death in Patients With Nonvalvular Atrial Fibrillation - The Hokuriku-Plus AF Registry -

Kenshi Hayashi, Toyonobu Tsuda, Akihiro Nomura, Noboru Fujino, Atsushi Nohara, Kenji Sakata, Tetsuo Konno, Chiaki Nakanishi, Hayato Tada, Yoji Nagata, Ryota Teramoto, Yoshihiro Tanaka, Masa-aki Kawashiri, Masakazu Yamagishi on behalf of the Hokuriku-Plus AF Registry Investigators

plastic Anemia and Risk of Incident Atrial Fibrillation - A Nationwide Cohort Study -

\section{Aplastic Anemia and Risk of Incident Atrial Fibrillatio
Wei-Syun Hu, Fung-Chang Sung, Cheng-Li Lin}

23. Comparison of the 2MACE and TIMI-AF Scores for Composite Clinical Outcomes in Anticoagulated Atrial Fibrillation Patients

Daniele Pastori, José Miguel Rivera-Caravaca, María Asunción Esteve-Pastor, Vanessa Roldán, Francisco Marín, Pasquale Pignatelli, Francesco Violi, Gregory Y.H. Lip

24. Safety and Efficacy of Subcutaneous Cardioverter Defibrillator in Patients at High Risk of Sudden Cardiac Death - Primary Japanese Experience -

Shingo Sasaki, Hirofumi Tomita, Takuo Tsurugi, Yuji Ishida, Yoshihiro Shoji, Kimitaka Nishizaki, Takahiko Kinjo, Tomohide Endo, Fumie Nishizaki, Kenji Hanada, Kenichi Sasaki, Daisuke Horiuchi, Masaomi Kimura, Takumi Higuma, Hideharu Okamatsu, Yasuaki Tanaka, Junjiroh Koyama, Ken Okumura

25. Periprocedural Complications in Patients Undergoing Catheter Ablation of Atrial Fibrillation Without Discontinuation of a Vitamin K Antagonist and Direct Oral Anticoagulants

Toshiya Kojima, Katsuhito Fujiu, Nobuaki Fukuma, Hiroshi Matsunaga, Tsukasa Oshima, Jun Matsuda, Takumi Matsubara, Yu Shimizu, Gaku Oguri, Eriko Hasumi, Hiroyuki Morita, Issei Komuro 
26. Low Risk of Pulmonary Vein Stenosis After Contemporary Atrial Fibrillation Ablation - Lessons From Repeat Procedures After Radiofrequency Current, Cryoballoon, and Laser Balloon -

Takahiko Nagase, Stefano Bordignon, Laura Perrotta, Fabrizio Bologna, Felix K. Weise, Athanasios Konstantinou, Ritsushi Kato, Boris Schmidt, Julian K. R. Chun

27. Sex Differences in Long-Term Clinical Outcomes in Patients With Atrial Fibrillation Undergoing Coronary Stent Implantation

Yukiko Matsumura-Nakano, Hiroki Shiomi, Takeshi Morimoto, Satoshi Shizuta, Kyohei Yamaji, Hirotoshi Watanabe, Yusuke Yoshikawa, Tomohiko Taniguchi, Tetsuma Kawaji, Masahiro Natsuaki, Takashi Akasaka, Keiichi Hanaoka, Kazushige Kadota, Ken Kozuma, Kengo Tanabe, Yoshihisa Nakagawa, Toshiya Muramatsu, Yoshihiro Morino, Kenji Ando, Takeshi Kimura

28. Metabolic Syndrome and the Risk of New-Onset Atrial Fibrillation in Middle-Aged East Asian Men Yong-Giun Kim, Kee-Joon Choi, Seungbong Han, Ki Won Hwang, Chang Hee Kwon, Gyung-Min Park, Ki-Bum Won, Soe Hee Ann, Jun Kim, Shin-Jae Kim, Sang-Gon Lee, Gi-Byoung Nam, You-Ho Kim

29. Treatment Strategies for Atrial Fibrillation With Left Ventricular Systolic Dysfunction - Meta-Analysis Jinhee Ahn, Hyun Jung Kim, Jeong Cheon Choe, Jin Sup Park, Hye Won Lee, Jun-Hyok Oh, Jung Hyun Choi, Han Cheol Lee, Kwang Soo Cha, Taek Jong Hong, Young-Hoon Kim

30. Effect of the Epicardial Adipose Tissue Volume on the Prevalence of Paroxysmal and Persistent Atrial Fibrillation Kageyuki Oba, Minetaka Maeda, Gulinu Maimaituxun, Satoshi Yamaguchi, Osamu Arasaki, Daiju Fukuda, Shusuke Yagi, Yukina Hirata, Susumu Nishio, Takashi Iwase, Shoichiro Takao, Kenya Kusunose, Hirotsugu Yamada, Takeshi Soeki, Tetsuzo Wakatsuki, Masafumi Harada, Hiroaki Masuzaki, Masataka Sata, Michio Shimabukuro

31. Effect of Atrial Fibrillation on the Incidence and Outcome of Osteoporotic Fracture - A Nationwide PopulationBased Study -

Daehoon Kim, Pil-Sung Yang, Tae-Hoon Kim, Jae-Sun Uhm, Junbeom Park, Hui-Nam Pak, Moon-Hyoung Lee, Boyoung Joung

32. Novel Ablation Strategy for Isolating the Superior Vena Cava Using Ultra High-Resolution Mapping Yasuaki Tanaka, Atsushi Takahashi, Takamitsu Takagi, Jun Nakajima, Katsumasa Takagi, Hiroyuki Hikita, Masahiko Goya, Kenzo Hirao

33. Clinical, Electrocardiographic, and Echocardiographic Parameter Combination Predicts the Onset of Atrial Fibrillation

Takeshi Soeki, Tomomi Matsuura, Takeshi Tobiume, Sachiko Bando, Kazuhisa Matsumoto, Hiromi Nagano, Etsuko Uematsu, Kenya Kusunose, Takayuki Ise, Koji Yamaguchi, Shusuke Yagi, Daiju Fukuda, Hirotsugu Yamada, Tetsuzo Wakatsuki, Michio Shimabukuro, Masataka Sata

34. Poor Rhythm Outcome of Catheter Ablation for Early-Onset Atrial Fibrillation in Women - Mechanistic Insight Hee Tae Yu, Pil-Sung Yang, Tae-Hoon Kim, Jae-Sun Uhm, Jong-Youn Kim, Boyoung Joung, Moon-Hyoung Lee, Hui-Nam Pak

35. Differential Diagnosis Between Catecholaminergic Polymorphic Ventricular Tachycardia and Long QT Syndrome Type 1 - Modified Schwartz Score Junichi Ozawa, Seiko Ohno, Yusuke Fujii, Takeru Makiyama, Hiroshi Suzuki, Akihiko Saitoh, Minoru Horie

36. Renal Dysfunction Affects Anticoagulation Control With Warfarin and Outcomes in Japanese Elderly Patients With Non-Valvular Atrial Fibrillation

Hiroshi Inoue, Eitaro Kodani, Hirotsugu Atarashi, Ken Okumura, Takeshi Yamashita, Hideki Origasa on behalf of the J-RHYTHM Registry Investigators

37. Impact of Pre-Existing Bradycardia on Subsequent Need for Pacemaker Implantation After Radiofrequency Catheter Ablation for Atrial Fibrillation

Tetsuma Kawaji, Satoshi Shizuta, Shintaro Yamagami, Takanori Aizawa, Takashi Yoshizawa, Masashi Kato, Takafumi Yokomatsu, Shinji Miki, Koh Ono, Takeshi Kimura

38. Three-Year Clinical Outcomes Associated With Warfarin vs. Direct Oral Anticoagulant Use Among Japanese Patients With Atrial Fibrillation - Findings From the SAKURA AF Registry Yasuo Okumura, Katsuaki Yokoyama, Naoya Matsumoto, Eizo Tachibana, Keiichiro Kuronuma, Koji Oiwa, Michiaki Matsumoto, Toshiaki Kojima, Shoji Hanada, Kazumiki Nomoto, Ken Arima, Fumiyuki Takahashi, Tomobumi Kotani, Yukitoshi Ikeya, Seiji Fukushima, Satoru Itou, Kunio Kondo, Masaaki Chiku, Yasumi Ohno, Motoyuki Onikura, Atsushi Hirayama for the SAKURA AF Registry Investigators

me in Therapeutic Range and Disease Outcomes in Elderly Japanese Patients With Nonvalvular Atrial Fibrillation Hiroshi Inoue, Eitaro Kodani, Hirotsugu Atarashi, Ken Okumura, Takeshi Yamashita, Yuji Okuyama, Hideki Origasa for the J-RHYTHM Registry Investigators

40. Clinical Manifestations and Long-Term Mortality in Lamin A/C Mutation Carriers From a Japanese Multicenter Registry

Kenzaburo Nakajima, Takeshi Aiba, Takeru Makiyama, Suguru Nishiuchi, Seiko Ohno, Koichi Kato, Yuta Yamamoto, Takahiro Doi, Satoshi Shizuta, Kenji Onoue, Nobue Yagihara, Taisuke Ishikawa, Ichiro Watanabe, Hiroshi Kawakami, Yasushi Oginosawa, Nobuyuki Murakoshi, Akihiko Nogami, Kazutaka Aonuma, Yoshihiko Saito, Takeshi Kimura, Satoshi Yasuda, Naomasa Makita, Wataru Shimizu, Minoru Horie, Kengo Kusano

41. Left Atrial Appendage Thrombus Prior to Atrial Fibrillation Ablation in the Era of Direct Oral Anticoagulants Masahide Harada, Masayuki Koshikawa, Yuji Motoike, Tomohide Ichikawa, Kunihiko Sugimoto, Eiichi Watanabe, Yukio Ozaki 
42. Ablation Index for Catheter Ablation of Atrial Fibrillation - Clinical Applicability and Comparison With ForceTime Integral -

Paula Münkler, Stefan Kröger, Spyridon Liosis, Amr Abdin, Evgeny Lyan, Charlotte Eitel, Ingo Eitel, Christian Meyer, Stephan Willems, Christian-Hendrik Heeger, Roland Richard Tilz

43. Uric Acid and Risk of Atrial Fibrillation in the Korean General Population Chang Hee Kwon, Sung Ho Lee, Jong-Young Lee, Seungho Ryu, Ki-Chul Sung

44. Relationship Between Anemia and the Risk of Sudden Cardiac Arrest - A Nationwide Cohort Study in South Korea -

In-Jung Kim, Pil-Sung Yang, Tae-Hoon Kim, Jae-Sun Uhm, Hui-Nam Pak, Moon-Hyoung Lee, Jung-Hoon Sung, Boyoung Joung

45. Validation of Conventional Thromboembolic Risk Factors in a Korean Atrial Fibrillation Population - Suggestion for a Novel Scoring System, $\mathrm{CHA}_{2} \mathrm{DS}_{2}-\mathrm{VAK}-$

Myung-Jin Cha, Youngjin Cho, Il-Young Oh, Eue-Keun Choi, Seil Oh

46. Defensive Implantable Cardioverter-Defibrillator Programming Is Safe and Reduces Inappropriate Therapy - Comparison of 3 Programming Strategies in 1,471 Patients -

Achim Leo Burger, Stefan Stojkovic, Herwig Schmidinger, Robin Ristl, Thomas Pezawas

47. Current Status, Time Trends and Outcomes of Combination Therapy With Oral Anticoagulant and Antiplatelet Drug in Patients With Atrial Fibrillation - The Fushimi AF Registry -

Nobutoyo Masunaga, Mitsuru Abe, Hisashi Ogawa, Yuya Aono, Syuhei Ikeda, Kosuke Doi, Yoshimori An, Mitsuru Ishii, Moritake Iguchi, Masahiro Esato, Hikari Tsuji, Hiromichi Wada, Koji Hasegawa, Gregory Y.H. Lip, Masaharu Akao on behalf of the Fushimi AF Registry Investigators

fety and Efficacy of Transvenous Lead Extraction With a High-Frequency Excimer Laser - A Single Center Experience -

Takakatsu Yoshitake, Masahiko Goya, Takeshi Sasaki, Shinya Shiohira, Masahiro Sekigawa, Yasuhiro Shirai, Kiko Lee, Atsuhiko Yagishita, Shingo Maeda, Yoshihide Takahashi, Mihoko Kawabata, Kenzo Hirao

Cardiovascular Intervention (21)

49. Percutaneous Left Atrial Appendage Closure With LACBES ${ }^{\circledR}$ Occluder - A Preclinical Feasibility Study Xuechao Tang, Zhigang Zhang, Feiyu Wang, Yuan Bai, Xudong Xu, Xinmiao Huang, Xianxian Zhao, Shanshi Gong, Yongwen Qin

50. Temporal Changes in Platelet Response in Acute Coronary Syndrome Patients With Prasugrel and Clopidogrel After Stent Implantation

Antonio Tello-Montoliu, José Rivera, Diana Hernández, Ana Silvente, Eva Jover, Ana I Rodriguez, Miriam Quintana, Ana Romero, Esteban Orenes-Piñero, José Miguel Rivera-Caravaca, Francisco Marín, Andrea Veliz, Mariano Valdés

51. Utilization of Anticoagulant and Antiplatelet Agents Among Patients With Atrial Fibrillation Undergoing Percutaneous Coronary Intervention - Retrospective Cohort Study Using a Nationwide Claims Database in Japan Fumiko Ono, Shiro Tanaka, Yoko M. Nakao, Koji Kawakami

52. Clinical Characteristics and Long-Term Outcomes of Rotational Atherectomy - J2T Multicenter Registry Iwao Okai, Tomotaka Dohi, Shinya Okazaki, Kentaro Jujo, Makoto Nakashima, Hisao Otsuki, Kazuki Tanaka, Hiroyuki Arashi, Ryuta Okabe, Fukuko Nagura, Yugo Nara, Hiroshi Tamura, Takeshi Kurata, Hideyuki Kawashima, Hiroyuki Kyono, Junichi Yamaguchi, Katsumi Miyauchi, Ken Kozuma, Nobuhisa Hagiwara, Hiroyuki Daida

53. Second-Generation vs. First-Generation Drug-Eluting Stents in Patients With Calcified Coronary Lesions - Pooled Analysis From the RESET and NEXT Trials -

Koji Nishida, Kenji Nakatsuma, Hiroki Shiomi, Masahiro Natsuaki, Kazuya Kawai, Takeshi Morimoto, Ken Kozuma, Keiichi Igarashi, Kazushige Kadota, Kengo Tanabe, Yoshihiro Morino, Kiyoshi Hibi, Takashi Akasaka, Mitsuru Abe, Satoru Suwa, Toshiya Muramatsu, Masakazu Kobayashi, Kazuoki Dai, Koichi Nakao, Yasuhiro Tarutani, Kenshi Fujii, Takeshi Kimura on behalf of the RESET and NEXT Investigators

54. Impact of Serum Phosphorus Levels on Outcomes After Implantation of Drug-Eluting Stents in Patients on Hemodialysis

Tatsuyuki Sato, Jiro Aoki, Ken Kozuma, Yasuyuki Maruyama, Kenya Nasu, Masaya Otsuka, Kenji Ando, Kiyoshi Hibi, Yoshiki Uehara, Kengo Tanabe, Yuji Ikari

55. Five-Year Clinical Outcomes After Drug-Eluting Stent Implantation Following Rotational Atherectomy for Heavily Calcified Lesions

Hiroyuki Jinnouchi, Shoichi Kuramitsu, Tomohiro Shinozaki, Takashi Hiromasa, Yohei Kobayashi, Yasuaki Takeji, Mizuki Miura, Hisaki Masuda, Yukiko Matsumura, Yuhei Yamaji, Kenichi Sakakura, Takenori Domei, Yoshimitsu Soga, Makoto Hyodo, Shinichi Shirai, Kenji Ando

56. Comparison of the Acute and Long-Term Outcomes of Patients With Multivessel Coronary Artery Disease After Angiographic Complete and Incomplete Revascularization With Drug-Eluting Stents I-Chang Hsieh, Ming-Jer Hsieh, Chun-Chi Chen, Chao-Yung Wang, Shang-Hung Chang, Cheng-Hung Lee, Dong-Yi Chen, Chia-Hung Yang, Ming-Lung Tsai 
57. Effect of Side Branch Predilation in Coronary Bifurcation Stenting With the Provisional Approach - Results From the COBIS (Coronary Bifurcation Stenting) II Registry -

Seung Hwa Lee, Young Bin Song, Joo Myung Lee, Taek Kyu Park, Jeong Hoon Yang, Joo-Yong Hahn, Jin-Ho Choi, Seung-Hyuk Choi, Sang Hoon Lee, Joong Hyun Ahn, Keumhee C. Carriere, Myung-Ho Jeong, Hyo-Soo Kim, Ju Hyeon Oh, Yangsoo Jang, Hyeon-Cheol Gwon

58. Randomized Prospective Comparison of Everolimus-Eluting vs. Sirolimus-Eluting Stents in Patients Undergoing Percutaneous Coronary Intervention - 3-Year Clinical Outcomes of the EXCELLENT Randomized Trial -

Kyung Woo Park, Tae-Min Rhee, Hyun-Jae Kang, Bon-Kwon Koo, Hyeon-Cheol Gwon, Jung-Han Yoon, Do-Sun Lim, In-Ho Chae, Kyoo-Rok Han, Taehoon Ahn, Myung-Ho Jeong, Dong-Woon Jeon, Yang-Soo Jang, Hyo-Soo Kim

59. Local Flow Patterns After Implantation of Bioresorbable Vascular Scaffold in Coronary Bifurcations - Novel Findings by Computational Fluid Dynamics -

Yingguang Li, Zehang Li, Emil N. Holck, Bo Xu, Antonios Karanasos, Zhenyu Fei, Yunxiao Chang, Miao Chu, Jouke Dijkstra, Evald H. Christiansen, Johan H. C. Reiber, Niels R. Holm, Shengxian Tu

60. Protective Effect of Remote Ischemic Preconditioning on Myocardial Damage After Percutaneous Coronary Intervention in Stable Angina Patients With Complex Coronary Lesions - Subanalysis of a Randomized Controlled Trial -

Kentaro Ejiri, Toru Miyoshi, Kunihisa Kohno, Makoto Nakahama, Masayuki Doi, Mitsuru Munemasa, Masaaki Murakami, Atsushi Takaishi, Kazufumi Nakamura, Hiroshi Ito for the RINC Study Collaborators

61. Comparison Between Long-Term Clinical Outcomes of Vitamin K Antagonist and Direct Oral Anticoagulants in Patients With Atrial Fibrillation Undergoing Percutaneous Coronary Intervention

Ruka Yoshida, Itsuro Morishima, Kensuke Takagi, Yasuhiro Morita, Hideyuki Tsuboi, Toyoaki Murohara

62. Clinical Outcomes of Drug-Coated Balloons in Coronary Artery Disease Unsuitable for Drug-Eluting Stent Implantation

Raisuke Iijima, Norihiro Kougame, Hidehiko Hara, Masao Moroi, Masato Nakamura

63. Intravascular Ultrasound and Angioscopy Assessment of Coronary Plaque Components in Chronic Totally Occluded Lesions

Shigeki Kimura, Tomoyo Sugiyama, Keiichi Hishikari, Shun Nakagama, Shun Nakamura, Toru Misawa, Masafumi Mizusawa, Kazuto Hayasaka, Yosuke Yamakami, Yuichiro Sagawa, Keisuke Kojima, Hirofumi Ohtani, Hiroyuki Hikita, Atsushi Takahashi

64. Non-Inferiority of Resolute Integrity Drug-Eluting Stent to Benchmark Xience Drug-Eluting Stent Masato Nakamura, Satoru Otsuji, Yoshihisa Nakagawa, Yuji Oikawa, Nobuo Shiode, Masatoshi Miyahara, Toshihito Furukawa, Gaku Nakazawa, Hiroyoshi Yokoi on behalf of the PROPEL Investigators

65. Atherosclerotic Plaque Component as a Risk Factor for Distal Embolization During Percutaneous Coronary Intervention - Pathology of Tissue Obtained by Distal Protection Device -

Akiko Fujino, Hiroyuki Hao, Sayaka Shimodai, Rika Kawakami, Koshi Matsuo, Yoshio Yasumura, Yoshiharu Higuchi, Masahiko Tsujimoto, Yasunori Ueda, Seiichi Hirota

66. Association of Subclinical Intrastent Thrombus Detected 9 Months After Implantation of 2nd-Generation DrugEluting Stent With Future Major Adverse Cardiac Events - A Coronary Angioscopic Study -

Shota Okuno, Takayuki Ishihara, Osamu Iida, Mitsutoshi Asai, Masaharu Masuda, Shin Okamoto, Kiyonori Nanto, Takashi Kanda, Takuya Tsujimura, Yasuhiro Matsuda, Mitsuyoshi Takahara, Masaaki Uematsu, Toshiaki Mano

67. Comparison of Transcarotid vs. Transfemoral Transcatheter Aortic Valve Implantation Masazumi Watanabe, Shinya Takahashi, Hironori Yamaoka, Taijiro Sueda, Antonio Piperata, Xavier Zirphile, Lionel Leroux, Julien Peltan, Louis Labrousse

68. Incidence and Clinical Course of Limb Dysfunction Post Cardiac Catheterization - A Systematic Review Muhammad Ayyaz Ul Haq, Muhammad Rashid, Ian C. Gilchrist, Olivier Bertrand, Chun Shing Kwok, Chun Wai Wong, Hossam M. Mansour, Yasser Baghdaddy, James Nolan, Maarten A.H. van Leeuwen, Mamas A. Mamas

69. Different Neointimal Pattern in Early vs. Late In-Stent Restenosis and Clinical Outcomes After Drug-Coated Balloon Angioplasty - An Optical Coherence Tomography Study -

Jung-Hee Lee, Hae Won Jung, Jung-Sun Kim, Sung-Jin Hong, Chul-Min Ahn, Byeong-Keuk Kim, Young-Guk Ko, Donghoon Choi, Myeong-Ki Hong, Yangsoo Jang

Cardiac Rehabilitation (3)

70. Nutritional Status and Energy Intake as Predictors of Functional Status After Cardiac Rehabilitation in Elderly Inpatients With Heart Failure - A Retrospective Cohort Study -

Satoshi Katano, Akiyoshi Hashimoto, Katsuhiko Ohori, Ayako Watanabe, Remi Honma, Rimi Yanase, Tomoyuki Ishigo, Takefumi Fujito, Hirofumi Ohnishi, Kazufumi Tsuchihashi, Sumio Ishiai, Tetsuji Miura

71. Need for Systematic Efforts to Modify Health-Related Behaviors After Acute Myocardial Infarction in Korea Seungwoo Cha, Jin Joo Park, Sunyoung Kim, Hong-Yup Ahn, Kyungdo Han, Yookyung Lee, Won-Seok Kim, Nam-Jong Paik 
72. Impact of High Respiratory Exchange Ratio During Submaximal Exercise on Adverse Clinical Outcome in Heart Failure

Naoya Kakutani, Arata Fukushima, Takashi Yokota, Takashi Katayama, Hideo Nambu, Ryosuke Shirakawa, Satoshi Maekawa, Takahiro Abe, Shingo Takada, Takaaki Furihata, Kota Ono, Koichi Okita, Shintaro Kinugawa, Toshihisa Anzai

Cardiovascular Surgery (19)

73. Effect of Recurrent Mitral Regurgitation After Mitral Valve Repair in Patients With Degenerative Mitral Regurgitation

Jung-Hwan Kim, Seung Hyun Lee, Hyun-Chel Joo, Young-Nam Youn, Kyung-Jong Yoo, Byung-Chul Chang, Sak Lee

74. Revascularization of Left Coronary System Using a Skeletonized Left Internal Mammary Artery - Sequential vs. Separate Grafting -

Qiang Ji, YunQing Shi, LiMin Xia, RunHua Ma, JinQiang Shen, Hao Lai, WenJun Ding, ChunSheng Wang

75. Analysis of Computational Fluid Dynamics and Particle Image Velocimetry Models of Distal-End Side-to-Side and End-to-Side Anastomoses for Coronary Artery Bypass Grafting in a Pulsatile Flow

Yoshiko Shintani, Kenji Iino, Yoshitaka Yamamoto, Hiroki Kato, Hirofumi Takemura, Takahiro Kiwata

76. Preoperative Assessment of Endothelial Function for Prediction of Adverse Events After Cardiovascular Surgery Yuichi Saito, Hideki Kitahara, Goro Matsumiya, Yoshio Kobayashi

77. Surgical Outcomes of Isolated Coronary Artery Bypass Grafting for Acute Coronary Syndrome - Based on the Japan Adult Cardiovascular Surgery Database -

Shunsuke Kawamoto, Hiroaki Miyata, Noboru Motomura, Kazuo Tanemoto, Shinichi Takamoto, Yoshikatsu Saiki

78. Clinical Implication of Transaortic Mitral Pannus Removal During Repeat Cardiac Surgery for Patients With Mechanical Mitral Valve Byungjoon Park, Kiick Sung, Pyo Won Park

79. Current Surgical Outcomes of Congenital Heart Surgery for Patients With Down Syndrome in Japan Takaya Hoashi, Norimichi Hirahara, Arata Murakami, Yasutaka Hirata, Hajime Ichikawa, Junjiro Kobayashi, Shinichi Takamoto

80. Clinical Results, Adverse Events, and Change in End-Organ Function in Elderly Patients With HeartMateII Left Ventricular Assist Device - Japanese Multicenter Study -

Daisuke Yoshioka, Koichi Toda, Minoru Ono, Takeshi Nakatani, Akira Shiose, Yoshiro Matsui, Kenji Yamazaki, Yoshikatsu Saiki, Akihiko Usui, Hiroshi Niinami, Goro Matsumiya, Hirokuni Arai, Yoshiki Sawa on behalf of the Japanese HeartMateII Investigators

81. Association Between Platelet Count and Postoperative Blood Loss in Patients Undergoing Cardiac Surgery With Cardiopulmonary Bypass and Fresh Frozen Plasma Administration Guided by Thromboelastometry

Junko Ichikawa, Yoshiko Osada, Mitsuharu Kodaka, Keiko Nishiyama, Makiko Komori

Evidence of Pulmonary Vascular Reverse Remodeling After Pulmonary Artery Banding Performed in Early Infancy in Patients With Congenital Heart Defects

Naoki Masaki, Yuriko Saiki, Masato Endo, Kay Maeda, Osamu Adachi, Masatoshi Akiyama, Shunsuke Kawamoto, Yoshikatsu Saiki

83. Can Tolvaptan Protect Renal Function in the Early Postoperative Period of Cardiac Surgery? - Results of a SingleCenter Randomized Controlled Study -

Yuichiro Kishimoto, Yoshinobu Nakamura, Shingo Harada, Takeshi Onohara, Satoru Kishimoto, Tomohiro Kurashiki, Yoshikazu Fujiwara, Motonobu Nishimura

84. Surgical Aortic Valve Replacement in Octogenerians in the Era of Transcatheter Aortic Valve Replacement Takashi Yamauchi, Takano Hiroshi, Koichi Toda, Yoshiki Sawa, Osaka Cardiovascular Surgery Research Group (OSCAR)

85. Propensity Score Matched Analysis of Mechanical vs. Bioprosthetic Valve Replacement in Patients With Previous Stroke

Shao-Wei Chen, Victor Chien-Chia Wu, Yu-Sheng Lin, Ching-Chang Chen, Dong-Yi Chen, Chih-Hsiang Chang, Pao-Hsien Chu, Pei-Chi Ting, An-Hsun Chou, Tien-Hsing Chen

86. Impact of Recurrent Ventricular Tachyarrhythmia on Outcome in Japanese Heart Transplant Candidates With a Left Ventricular Assist Device

Hidetoshi Hattori, Atsushi Suzuki, Tsuyoshi Shiga, Tomohiro Nishinaka, Satoshi Saito, Kenji Yamazaki, Shinichi Nunoda, Nobuhisa Hagiwara

87. Safe Use of Glutaraldehyde to Repair the Destroyed Valve in Active Infective Mitral Valve Endocarditis

Ken Nakamura, Kazuhiro Hashimoto, Yoshimasa Sakamoto, Ko Bando, Michio Yoshitake, Yoko Matsumura, Katsushi Kinouchi, Takayuki Abe

88. Clinical Outcomes of Repeat Aortic Valve Replacement for Subaortic Pannus in Mechanical Aortic Valve Pyo Won Park, Byungjoon Park, Dong Seop Jeong, Kiick Sung, Wook Sung Kim, Young Tak Lee, Seung Woo Park

89. Robotic Transmitral Approach for Hypertrophic Cardiomyopathy With Systolic Anterior Motion Nai-Kuan Chou, Ryoi Okano, Takeo Tedoriya, I-Hui Wu, Hsi-Yu Yu, Yih-Sharng Chen, Ming-Jiuh Wang, Nai-Hsin Chi 
90. Trifecta vs. Magna for Aortic Valve Replacement - Differences in Clinical Outcome and Valve Hemodynamics Naoki Tadokoro, Satsuki Fukushima, Yusuke Shimahara, Yorihiko Matsumoto, Kizuku Yamashita, Naonori Kawamoto, Kimito Minami, Junjiro Kobayashi, Tomoyuki Fujita

91. Strategy of Cardiovascular Surgery for Patients With Dementia as Evaluated by Mini-Mental State Examination Sachie Terazawa, Hideki Oshima, Yuji Narita, Kazuro Fujimoto, Masato Mutsuga, Yoshiyuki Tokuda, Tomo Yoshizumi, Hideki Ito, Wataru Uchida, Akihiko Usui

Epidemiology (20)

92. Impact of Speed-Eating Habit on Subsequent Body Mass Index and Blood Pressure Among Schoolchildren - The Ibaraki Children's Cohort Study (IBACHIL) -

Kazumasa Yamagishi, Toshimi Sairenchi, Nobuyuki Sawada, Keiko Sunou, Mizuki Sata, Utako Murai, Nobue Takizawa, Fujiko Irie, Hiroshi Watanabe, Hiroyasu Iso, Hitoshi Ota

93. Time Trend in Interest and Satisfaction Towards Clinical Training and Academic Activities Among Early-Career Cardiologists - The Japanese Circulation Society Post-Graduate Training Survey -

Takashi Kohno, Shun Kohsaka, Yasuyoshi Takei, Keiichi Fukuda, Yukio Ozaki, Akira Yamashina

94. Metabolic Syndrome Over 4 Years Before the Onset of Cardiovascular Disease - Nested Case-Control Study Huanhuan Hu, Tohru Nakagawa, Toru Honda, Shuichiro Yamamoto, Akiko Nanri, Maki Konishi, Hiroko Okazaki, Keisuke Kuwahara, Ai Hori, Chihiro Nishiura, Ikuko Kashino, Teppei Imai, Akiko Nishihara, Shamima Akter, Toshiaki Miyamoto, Naoko Sasaki, Takayuki Ogasawara, Akihiko Uehara, Makoto Yamamoto, Taizo Murakami, Makiko Shimizu, Masafumi Eguchi, Takeshi Kochi, Satsue Nagahama, Kentaro Tomita, Isamu Kabe, Tetsuya Mizoue, Tomofumi Sone, Seitaro Dohi for the Japan Epidemiology Collaboration on Occupational Health Study Group

95. Treatment Patterns, Statin Intolerance, and Subsequent Cardiovascular Events Among Japanese Patients With High Cardiovascular Risk Initiating Statin Therapy

Saurabh P. Nagar, Pratik P. Rane, Kathleen M. Fox, Juliana Meyers, Keith Davis, Anne Beaubrun, Hyoe Inomata, Yi Qian, Kouji Kajinami

96. Ability of B-Type Natriuretic Peptide Testing to Predict Cardioembolic Stroke in the General Population - Comparisons With C-Reactive Protein and Urinary Albumin -

Motoyuki Nakamura, Yasuhiro Ishibashi, Fumitaka Tanaka, Shinichi Omama, Toshiyuki Onoda, Tomohiro Takahashi, Shuko Takahashi, Kozo Tanno, Masaki Ohsawa, Kiyomi Sakata, Makoto Koshiyama, Kuniaki Ogasawara, Akira Okayama on behalf of the Iwate-KENCO Study Group

97. Epidemiology of Pediatric Out-of-Hospital Cardiac Arrest at School - An Investigation of a Nationwide Registry in Japan -

Kosuke Kiyohara, Junya Sado, Tetsuhisa Kitamura, Mamoru Ayusawa, Masahiko Nitta, Taku Iwami, Ken Nakata, Yasuto Sato, Noriko Kojimahara, Naohito Yamaguchi, Tomotaka Sobue, Yuri Kitamura for the SPIRITS Investigators

98. "Ikigai”, Subjective Wellbeing, as a Modifier of the Parity-Cardiovascular Mortality Association - The Japan Collaborative Cohort Study -

Sumiyo Yasukawa, Eri Eguchi, Keiki Ogino, Akiko Tamakoshi, Hiroyasu Iso

9. Nonfasting Glucose and Incident Stroke and Its Types - The Circulatory Risk in Communities Study (CIRCS) -

Hironori Imano, Hiroyasu Iso, Akihiko Kitamura, Kazumasa Yamagishi, Mina Hayama-Terada, Isao Muraki, Takeo Okada, Mitsumasa Umesawa, Tetsuya Ohira, Tomoko Sankai, Renzhe Cui, Takeshi Tanigawa, Masahiko Kiyama on behalf of the CIRCS Investigators

100. Low-Density Lipoprotein Cholesterol Goal Attainment Rates by Initial Statin Monotherapy Among Patients With Dyslipidemia and High Cardiovascular Risk in Japan - A Retrospective Database Analysis -

Tatsuya Umeda, Ai Hayashi, Akiko Harada, Kotoba Okuyama, Carl A. Baxter, Shigeru Tokita, Tamio Teramoto

101. Minor Electrocardiographic ST-T Change and Risk of Stroke in the General Japanese Population Joji Ishikawa, Hideo Hirose, Joseph E Schwartz, Shizukiyo Ishikawa

Comparing the Consistency and Performance of Various Coronary Heart Disease Prediction Models for Primary Prevention Using a National Representative Cohort in Taiwan

Kuo-Liong Chien, Hung-Ju Lin, Ta-Chen Su, Yun-Yu Chen, Pei-Chun Chen

103. Association of N-Terminal Pro B-Type Natriuretic Peptide With Blood Pressure and Pulse Pressure in Elderly People - A Cross-Sectional Population Study -

Nobuo Sasaki, Hideya Yamamoto, Ryoji Ozono, Saeko Fujiwara, Yasuki Kihara

104. N-Terminal Pro-B-Type Natriuretic Peptide Is Not a Significant Predictor of Stroke Incidence After 5 Years - The Ohasama Study -

Michihiro Satoh, Takahisa Murakami, Kei Asayama, Takuo Hirose, Masahiro Kikuya, Ryusuke Inoue, Megumi Tsubota-Utsugi, Keiko Murakami, Ayako Matsuda, Azusa Hara, Taku Obara, Ryo Kawasaki, Kyoko Nomura, Hirohito Metoki, Koichi Node, Yutaka Imai, Takayoshi Ohkubo

inh Intake and Death From Pulmonary Embolisms Among Japanese Men and Women - The Japan Collaborative Cohort (JACC) Study -

Tetsuya Ohira, Hiroyasu Iso, Kazumasa Yamagishi, Akiko Tamakoshi, JACC Study Group 
106. Change in Pericardial Fat Volume and Cardiovascular Risk Factors in a General Population of Japanese Men Itsuko Miyazawa, Takayoshi Ohkubo, Sayaka Kadowaki, Akira Fujiyoshi, Takashi Hisamatsu, Aya Kadota, Hisatomi Arima, Matthew Budoff, Kiyoshi Murata, Katsuyuki Miura, Hiroshi Maegawa, Hirotsugu Ueshima for the SESSA Research Group

107. Two-Year Survival After First-Ever Stroke in a General Population of 1.4 Million Japanese - Shiga Stroke Registry Naoyuki Takashima, Hisatomi Arima, Yoshikuni Kita, Takako Fujii, Naomi Miyamatsu, Masaru Komori, Yoshihisa Sugimoto, Satoru Nagata, Katsuyuki Miura, Kazuhiko Nozaki

108. Associations of High-Density Lipoprotein Particle and High-Density Lipoprotein Cholesterol With Alcohol Intake, Smoking, and Body Mass Index - The INTERLIPID Study -

Maryam Zaid, Katsuyuki Miura, Akira Okayama, Hideaki Nakagawa, Kiyomi Sakata, Shigeyuki Saitoh, Nagako Okuda, Katsushi Yoshita, Sohel R. Choudhury, Beatriz Rodriguez, Kamal Masaki, Bradley Willcox, Naoko Miyagawa, Tomonori Okamura, Queenie Chan, Paul Elliott, Jeremiah Stamler, Hirotsugu Ueshima for the INTERLIPID and INTERMAP Research Groups

109. Effect of Hypertension on Aortic Artery Disease-Related Mortality - 3.8-Year Nationwide Community-Based Prospective Cohort Study -

Yoichiro Otaki, Tetsu Watanabe, Tsuneo Konta, Masafumi Watanabe, Shouichi Fujimoto, Yuji Sato, Koichi Asahi, Kunihiro Yamagata, Kazuhiko Tsuruya, Ichiei Narita, Masato Kasahara, Yugo Shibagaki, Kunitoshi Iseki, Toshiki Moriyama, Masahide Kondo, Tsuyoshi Watanabe

110. Smoking, Smoking Cessation, and Risk of Mortality in a Japanese Working Population - Japan Epidemiology Collaboration on Occupational Health Study -

Shamima Akter, Tohru Nakagawa, Toru Honda, Shuichiro Yamamoto, Keisuke Kuwahara, Hiroko Okazaki, Huanhuan Hu, Teppei Imai, Akiko Nishihara, Toshiaki Miyamoto, Naoko Sasaki, Takayuki Ogasawara, Akihiko Uehara, Makoto Yamamoto, Taizo Murakami, Makiko Shimizu, Masafumi Eguchi, Takeshi Kochi, Ai Hori, Satsue Nagahama, Kentaro Tomita, Maki Konishi, Ikuko Kashino, Akiko Nanri, Isamu Kabe, Tetsuya Mizoue, Naoki Kunugita, Seitaro Dohi for the Japan Epidemiology Collaboration on Occupational Health Study Group

111. Serum Fatty Acid and Risk of Coronary Artery Disease - Circulatory Risk in Communities Study (CIRCS) Choy-Lye Chei, Kazumasa Yamagishi, Akihiko Kitamura, Masahiko Kiyama, Tomoko Sankai, Takeo Okada, Hironori Imano, Tetsuya Ohira, Renzhe Cui, Mitsumasa Umesawa, Isao Muraki, Takeshi Tanigawa, Shinichi Sato, Hiroyasu Iso for the CIRCS Investigators

Heart Failure (39)

112. Effect of Mitral Valve Surgery in Patients With Dilated Cardiomyopathy and Severe Functional Mitral Regurgitation Hyemoon Chung, Makoto Amaki, Seiji Takashio, Hiroyuki Takahama, Takahiro Ohara, Takuya Hasegawa, Yasuo Sugano, Tomoyuki Fujita, Junjiro Kobayashi, Masanori Asakura, Hideaki Kanzaki, Toshihisa Anzai, Masafumi Kitakaze

113. Efficacy and Safety of Switching From Oral Bisoprolol to Transdermal Patch in Japanese Patients With Chronic Heart Failure

Shin-ichi Momomura, Yoshihiko Saito, Yoshio Yasumura, Kazuhiro Yamamoto, Yasushi Sakata, Masao Daimon, Koichiro Kinugawa, Hiroshi Okamoto, Naoki Dohi, Issei Komuro

114. Double-Blind, Randomized, Placebo-Controlled Trial Evaluating the Efficacy and Safety of Eplerenone in Japanese Patients With Chronic Heart Failure (J-EMPHASIS-HF)

Hiroyuki Tsutsui, Hiroshi Ito, Masafumi Kitakaze, Issei Komuro, Toyoaki Murohara, Tohru Izumi, Kenji Sunagawa, Yoshio Yasumura, Masafumi Yano, Kazuhiro Yamamoto, Tsutomu Yoshikawa, Takayoshi Tsutamoto, Junwei Zhang, Akifumi Okayama, Yoshihiko Ichikawa, Kazuhiro Kanmuri, Masunori Matsuzaki for the J-EMPHASIS-HF Study Group

115. Effects of Additive Tolvaptan vs. Increased Furosemide on Heart Failure With Diuretic Resistance and Renal Impairment - Results From the K-STAR Study -

Takayuki Inomata, Yuki Ikeda, Keisuke Kida, Yugo Shibagaki, Naoki Sato, Yuji Kumagai, Hisahito Shinagawa, Junya Ako, Tohru Izumi for the Kanagawa Aquaresis Investigators

116. Circulating ANGPTL2 Levels Increase in Humans and Mice Exhibiting Cardiac Dysfunction

Zhe Tian, Keishi Miyata, Jun Morinaga, Haruki Horiguchi, Tsuyoshi Kadomatsu, Motoyoshi Endo, Jiabin Zhao, Shunshun Zhu, Taichi Sugizaki, Michio Sato, Kazutoyo Terada, Takahiro Okumura, Toyoaki Murohara, Yuichi Oike

117. Relationship Between Bacteremia and Hemorrhagic Stroke in Patients With Continuous-Flow Left Ventricular Assist Device

Daisuke Yoshioka, Ryoto Sakaniwa, Koichi Toda, Takaaki Samura, Shunsuke Saito, Noriyuki Kashiyama, Shuhei Okazaki, Keitaro Domae, Yasushi Yoshikawa, Shigeru Miyagawa, Hiroyasu Iso, Yasushi Sakata, Yoshiki Sawa

118. Anemia Is Associated With Blunted Response to $\beta$-Blocker Therapy Using Carvedilol - Insights From Japanese Chronic Heart Failure (J-CHF) Study -

Yuji Nagatomo, Tsutomu Yoshikawa, Hiroshi Okamoto, Akira Kitabatake, Masatsugu Hori on behalf of J-CHF Investigators 
119. Early Prediction Model for Successful Bridge to Recovery in Patients With Fulminant Myocarditis Supported With Percutaneous Venoarterial Extracorporeal Membrane Oxygenation - Insights From the CHANGE PUMP Study Akinori Sawamura, Takahiro Okumura, Akihiro Hirakawa, Masaaki Ito, Yukio Ozaki, Nobuyuki Ohte, Tetsuya Amano, Toyoaki Murohara on behalf of the CHANGE PUMP Investigators

120. Association of Age at Menarche With Left Ventricular Diastolic Dysfunction in Middle-Aged Women Seungho Ryu, Yoosoo Chang, Jeong Gyu Kang, Jidong Sung, Jang-Young Kim, Hyun-Suk Jung, Kyung Eun Yun, Chan-Won Kim, Juhee Cho, Min-Jung Kwon, Kye-Hyun Kim, Hocheol Shin, Ki-Chul Sung

121. In-Depth Insight Into the Mechanisms of Cardiac Dysfunction in Patients With Childhood Cancer After Anthracycline Treatment Using Layer-Specific Strain Analysis

Kana Yazaki, Ken Takahashi, Sachie Shigemitsu, Mariko Yamada, Takeshi Iso, Maki Kobayashi, Katsumi Akimoto, Hiroyuki Tamaichi, Junya Fujimura, Masahiro Saito, Masaki Nii, Toshiaki Shimizu

122. Applying the Seattle Heart Failure Model in the Office Setting in the Era of Electronic Medical Records Brent A. Williams, Shikhar Agarwal

123. Left Ventricular Mass Influences Relationship Between Filling Pressure and Early-Diastolic Ratio of Inflow Velocity to Mitral Annular Velocity (E/e')

Masahiro Nakabachi, Satoshi Yamada, Hiroyuki Iwano, Taichi Hayashi, Kazunori Okada, Kenya Kusunose, Kiyotaka Watanabe, Tomoko Ishizu, Kazuaki Wakami, Hirotsugu Yamada, Kaoru Dohi, Yoshihiro Seo, Nobuyuki Ohte, Chikara Shimizu, Taisei Mikami

124. Skeletal Muscle Pump Function Is Associated With Exercise Capacity in Patients With Heart Failure Toru Kondo, Sumio Yamada, Chikako Asai, Takahiro Okumura, Daisuke Tanimura, Toyoaki Murohara

125. Serial Change in Serum Chloride During Hospitalization Could Predict Heart Failure Death in Acute Decompensated Heart Failure Patients

Takumi Kondo, Takahisa Yamada, Shunsuke Tamaki, Takashi Morita, Yoshio Furukawa, Yusuke Iwasaki, Masato Kawasaki, Atsushi Kikuchi, Tatsuhisa Ozaki, Yoshihiro Sato, Masahiro Seo, Iyo Ikeda, Eiji Fukuhara, Makoto Abe, Jun Nakamura, Yasushi Sakata, Masatake Fukunami

126. Survival of HeartMate II Patients Despite Cessation of Anticoagulation - Outcomes and Hemostatic Analysis Rashad Zayat, Mohammad Amen Khattab, Oliver Grottke, Markus Honickel, Andreas Goetzenich, Ajay Moza, Christian Stoppe, Rüdiger Autschbach, Lachmandath Tewarie

127. Endogenous Adenosine May Be Related to Left Ventricular Dysfunction, Dilation, and Wall Thinning in Patients With Heart Disease

Yujiro Kinomura, Toshiki Tanaka, Takuma Aoyama, Masanori Hatasa, Shingo Minatoguchi, Masamitsu Iwasa, Yoshihisa Yamada, Takahide Nawa, Hiromitsu Kanamori, Masanori Kawasaki, Yukihiro Esaka, Bunji Uno, Kazuhiko Nishigaki, Shinya Minatoguchi

128. Incidence and Risk Factors of Stroke or Systemic Embolism in Patients With Atrial Fibrillation and Heart Failure - The Fushimi AF Registry -

Moritake Iguchi, Yuji Tezuka, Hisashi Ogawa, Yasuhiro Hamatani, Daisuke Takagi, Yoshimori An, Takashi Unoki, Mitsuru Ishii, Nobutoyo Masunaga, Masahiro Esato, Hikari Tsuji, Hiromichi Wada, Koji Hasegawa, Mitsuru Abe, Gregory Y.H. Lip, Masaharu Akao

129. Nationwide Survey of Palliative Care for Patients With Heart Failure in Japan

Takashi Kuragaichi, Yuma Kurozumi, Shogo Ohishi, Yasuo Sugano, Akihiro Sakashita, Norihiko Kotooka, Makoto Suzuki, Taiki Higo, Dai Yumino, Yasuko Takada, Seiko Maeda, Saori Yamabe, Koichi Washida, Tomonori Takahashi, Tomohito Ohtani, Yasushi Sakata, Yukihito Sato

130. Novel Risk Score Efficiently Prevents Tolvaptan-Induced Hypernatremic Events in Patients With Heart Failure Koichiro Kinugawa, Naoki Sato, Takayuki Inomata, Moriyoshi Yasuda, Yoshiyuki Shibasaki, Toshiyuki Shimakawa

131. Donor Polymorphisms in Genes Related to B-Cell Biology Associated With Antibody-Mediated Rejection After Heart Transplantation

Grecia M. Marrón-Liñares, Lucía Núñez, María G. Crespo-Leiro, Eloy Álvarez-López, Eduardo Barge-Caballero, Gonzalo Barge-Caballero, David Couto-Mallón, Concepción Pradas-Irun, Javier Muñiz, Carmela Tan, E. Rene Rodríguez, José Manuel Vázquez-Rodríguez, Manuel Hermida-Prieto

132. Geriatric Nutritional Risk Index Predicts Cardiovascular Events in Patients at Risk for Heart Failure Masatoshi Minamisawa, Takashi Miura, Hirohiko Motoki, Yasushi Ueki, Hitoshi Nishimura, Kunihiko Shimizu, Wataru Shoin, Mikiko Harada, Tomoaki Mochidome, Keisuke Senda, Koji Yoshie, Yasutaka Oguchi, Naoto Hashizume, Naoyuki Abe, Tatsuya Saigusa, Soichiro Ebisawa, Atsushi Izawa, Jun Koyama, Uichi Ikeda, Koichiro Kuwahara

133. The Unraveled Link Between Antiviral Therapy and Heart Failure Hospitalization in Chronic Hepatitis C Virus Infection - A Nationwide Cohort Study -

Ming-Shyan Lin, Chang-Min Chung, Ming-Ling Chang, Mei-Yen Chen, Shih-Tai Chang, Pao-Hsien Chu, Tien-Hsing Chen, Wey-Yil Lin, Tung-Jung Huang, Yu-Sheng Lin

134. Routine Indwelling Urethral Catheterization in Acute Heart Failure Patients Is Associated With Increased Urinary Tract Complications Without Improved Heart Failure Outcomes Albert Youngwoo Jang, Connor O’Brien, Wook-Jin Chung, Pyung Chun Oh, Jongwook Yu, Kyounghoon Lee, Woong Chol Kang, Jeonggeun Moon 
135. Non-Ischemic Heart Failure With Reduced Ejection Fraction Is Associated With Altered Intestinal Microbiota Themistoklis Katsimichas, Tomohito Ohtani, Daisuke Motooka, Yasumasa Tsukamoto, Hidetaka Kioka, Kei Nakamoto, Shozo Konishi, Misato Chimura, Kaoruko Sengoku, Hiroshi Miyawaki, Taiki Sakaguchi, Ryu Okumura, Konstantinos Theofilis, Tetsuya Iida, Kiyoshi Takeda, Shota Nakamura, Yasushi Sakata

136. Impacts of Predischarge Diastolic Functional Recovery on Clinical Outcomes in Patients With Hypertensive Heart Failure

Hyun Ju Yoon, Kye Hun Kim, Hyukjin Park, Jae Yeong Cho, Young Joon Hong, Hyung Wook Park, Ju Han Kim, Youngkeun Ahn, Myung Ho Jeong, Jeong Gwan Cho, Jong Chun Park

137. Effect of QRS Morphology and Duration on Clinical Outcomes After Cardiac Resynchronization Therapy - Analysis of Japanese Multicenter Registry -

Takafumi Oka, Koichi Inoue, Koji Tanaka, Yuko Hirao, Takaaki Isshiki, Takeshi Kimura, Masakiyo Nobuyoshi, Satoshi Shizuta, Takeshi Arita, Satoki Fujii, Katsuomi Iwakura, Kenshi Fujii, Kenji Ando

138. Prognostic Relevance of Liver Stiffness Assessed by Transient Elastography in Patients With Acute Decompensated Heart Failure

Yuki Saito, Mahoto Kato, Koichi Nagashima, Koyuru Monno, Yoshihiro Aizawa, Yasuo Okumura, Naoki Matsumoto, Mitsuhiko Moriyama, Atsushi Hirayama

139. Fate of Acute Heart Failure Patients With Mid-Range Ejection Fraction

Hye Bin Gwag, Ga Yeon Lee, Jin-Oh Choi, Hae-Young Lee, Jae-Joong Kim, Kyung-Kuk Hwang, Shung Chull Chae, Sang Hong Baek, Seok-Min Kang, Dong-Ju Choi, Byung-Su Yoo, Kye Hun Kim, Hyun-Young Park, Myeong-Chan Cho, Byung-Hee Oh, Eun-Seok Jeon on behalf of the KorAHF Registry

140. Effects of Carperitide on Degree of Pulmonary Congestion in Treatment of Acute Heart Failure Yuichi Kawase, Reo Hata, Takeshi Tada, Harumi Katoh, Kazushige Kadota

141. Prognostic Value of Electrocardiography in Patients With Fulminant Myocarditis Supported by Percutaneous Venoarterial Extracorporeal Membrane Oxygenation - Analysis From the CHANGE PUMP Study Akinori Sawamura, Takahiro Okumura, Masaaki Ito, Yukio Ozaki, Nobuyuki Ohte, Tetsuya Amano, Toyoaki Murohara on behalf of the CHANGE PUMP Investigators

142. Sex Differences in the Prognostic Power of Brain Natriuretic Peptide and N-Terminal Pro-Brain Natriuretic Peptide for Cardiovascular Events - The Japan Morning Surge-Home Blood Pressure Study -

Mizuri Taki, Yusuke Ishiyama, Hiroyuki Mizuno, Takahiro Komori, Ken Kono, Satoshi Hoshide, Kazuomi Kario

143. Correlates and Prognostic Values of Appearance of L Wave in Heart Failure Patients With Preserved vs. Reduced Ejection Fraction

Kumiko Masai, Toshiaki Mano, Akiko Goda, Masataka Sugahara, Aika Daimon, Masanori Asakura, Masaharu Ishihara, Tohru Masuyama

144. Baseline Global Longitudinal Strain as a Predictor of Left Ventricular Dysfunction and Hospitalization for Heart Failure of Patients With Malignant Lymphoma After Anthracycline Therapy

Keiko Hatazawa, Hidekazu Tanaka, Akiko Nonaka, Hiroki Takada, Fumitaka Soga, Yutaka Hatani, Hiroki Matsuzoe, Hiroyuki Shimoura, Junichi Ooka, Hiroyuki Sano, Yasuhide Mochizuki, Kensuke Matsumoto, Ken-ichi Hirata

145. Angiotensin Receptor Neprilysin Inhibitor in Japanese Patients With Heart Failure and Reduced Ejection Fraction - Baseline Characteristics and Treatment of PARALLEL-HF Trial -

Hiroyuki Tsutsui, Shin-ichi Momomura, Yoshihiko Saito, Hiroshi Ito, Kazuhiro Yamamoto, Tomomi Ohishi, Naoko Okino, Toshihito Kitamura, Weinong Guo

146. LINC-PINT Activates the Mitogen-Activated Protein Kinase Pathway to Promote Acute Myocardial Infarction by Regulating miR-208a-3p

Jianzhong Zhu, Huimin Gu, Xiaolei Lv, Chunying Yuan, Ping Ni, Feng Liu

147. Prognostic Impact of the Preservation of Activities of Daily Living on Post-Discharge Outcomes in Patients With Acute Heart Failure

Yusuke Uemura, Rei Shibata, Kenji Takemoto, Masayoshi Koyasu, Shinji Ishikawa, Toyoaki Murohara, Masato Watarai

148. Transcatheter Repair of Functional Mitral Regurgitation in Heart Failure Patients - A Meta-Analysis of 23 Studies on MitraClip Implantation -

Roberta De Rosa, Angelo Silverio, Cesare Baldi, Marco Di Maio, Costantina Prota, Ilaria Radano, Julia Rey, Eva Herrmann, Rodolfo Citro, Federico Piscione, Gennaro Galasso

149. Demographics, Management, and In-Hospital Outcome of Hospitalized Acute Heart Failure Syndrome Patients in Contemporary Real Clinical Practice in Japan - Observations From the Prospective, Multicenter Kyoto Congestive Heart Failure (KCHF) Registry -

Hidenori Yaku, Neiko Ozasa, Takeshi Morimoto, Yasutaka Inuzuka, Yodo Tamaki, Erika Yamamoto, Yusuke Yoshikawa, Takeshi Kitai, Ryoji Taniguchi, Moritake Iguchi, Masashi Kato, Mamoru Takahashi, Toshikazu Jinnai, Tomoyuki Ikeda, Kazuya Nagao, Takafumi Kawai, Akihiro Komasa, Ryusuke Nishikawa, Yuichi Kawase, Takashi Morinaga, Kanae Su, Mitsunori Kawato, Kenichi Sasaki, Mamoru Toyofuku, Yutaka Furukawa, Yoshihisa Nakagawa, Kenji Ando, Kazushige Kadota, Satoshi Shizuta, Koh Ono, Yukihito Sato, Koichiro Kuwahara, Takao Kato, Takeshi Kimura on behalf of the KCHF Study Investigators 
150. Impact of Coronary Artery Calcification in the Donor Heart on Transmitted Coronary Artery Disease in Heart Transplant Recipients

Yuki Kimura, Osamu Seguchi, Keiichiro Iwasaki, Koichi Toda, Noriko Kikuchi, Sachi Matsuda, Yuto Kumai, Kensuke Kuroda, Kyoichi Wada, Yorihiko Matsumoto, Satsuki Fukushima, Masanobu Yanase, Tomoyuki Fujita, Junjiro Kobayashi, Norihide Fukushima

Hypertension and Circulatory Control (4)

151. Csk Regulates Blood Pressure by Controlling the Synthetic Pathways of Aldosterone Sung-Moon Kim, Ji-One Kang, Ji Eun Lim, Sue-Yun Hwang, Bermseok Oh

152. Effects of Calcium-Channel Blocker Benidipine-Based Combination Therapy on Cardiac Events - Subanalysis of the COPE Trial -

Seiji Umemoto, Toshio Ogihara, Masunori Matsuzaki, Hiromi Rakugi, Kazuyuki Shimada, Masatoshi Kawana, Kazuomi Kario, Yasuo Ohashi, Takao Saruta for the Combination Therapy of Hypertension to Prevent Cardiovascular Events (COPE) Trial Group

153. Questionnaire Survey From the Viewpoint of Concordance in Patient and Physician Satisfaction Concerning Hypertensive Treatment in Elderly Patients - Patients Voice Study -

Yoshiyuki Ikeda, Takeshi Sasaki, So Kuwahata, Masakazu Imamura, Kanyo Tanoue, Shinichirou Komaki, Mamoru Hashiguchi, Atsushi Kuroda, Yuichi Akasaki, Chikuma Hamada, Mitsuru Ohishi

154. Relationship Between Cotinine-Verified Smoking Status and Incidence of Hypertension in 74,743 Korean Adults Byung Jin Kim, Dae Chul Seo, Bum Soo Kim, Jin Ho Kang

Imaging (14)

155. Morphological Changes and Device Migration After Stent Graft Insertion - Clinical Application of an Image-Based Modeling System and Analysis With Geometric Parameters Youkou Nemoto, Katsuyuki Hoshina, Masaharu Kobayashi, Masaru Kimura, Sota Yamamoto, Toshiaki Watanabe, Marie Ohshima

156. Early Diagnosis of Infective Endocarditis by Brain T2*-Weighted Magnetic Resonance Imaging Takashi Fujimoto, Yoichi Morofuji, Yuki Matsunaga, Nobutaka Horie, Tsuyoshi Izumo, Yohei Tateishi, Minoru Morikawa, Takashi Miura, Kiyoyuki Eishi, Takayuki Matsuo

157. Increased Prognostic Value of Query Amyloid Late Enhancement Score in Light-Chain Cardiac Amyloidosis Ke Wan, Jiayu Sun, Yuchi Han, Hong Liu, Dan Yang, Weihao Li, Jie Wang, Wei Cheng, Qing Zhang, Zhi Zeng, Yucheng Chen

158. High Signal Intensity on T2-Weighted Cardiovascular Magnetic Resonance Imaging Predicts Life-Threatening Arrhythmic Events in Hypertrophic Cardiomyopathy Patients

Yasuki Hen, Ayako Takara, Nobuo Iguchi, Yuko Utanohara, Kunihiko Teraoka, Kaori Takada, Haruhiko Machida, Itaru Takamisawa, Morimasa Takayama, Tsutomu Yoshikawa

159. Current Use of Intracoronary Imaging in Interventional Practice - Results of a European Association of Percutaneous Cardiovascular Interventions (EAPCI) and Japanese Association of Cardiovascular Interventions and Therapeutics (CVIT) Clinical Practice Survey -

Konstantinos C. Koskinas, Masato Nakamura, Lorenz Räber, Roisin Colleran, Kazushige Kadota, Davide Capodanno, William Wijns, Takashi Akasaka, Marco Valgimigli, Giulio Guagliumi, Stephan Windecker, Robert A. Byrne

160. Local Thickness of Epicardial Adipose Tissue Surrounding the Left Anterior Descending Artery Is a Simple Predictor of Coronary Artery Disease - New Prediction Model in Combination With Framingham Risk Score -

Gulinu Maimaituxun, Michio Shimabukuro, Daiju Fukuda, Shusuke Yagi, Yukina Hirata, Takashi Iwase, Shoichiro Takao, Tomomi Matsuura, Takayuki Ise, Kenya Kusunose, Takeshi Tobiume, Koji Yamaguchi, Hirotsugu Yamada, Takeshi Soeki, Tetsuzo Wakatsuki, Masafumi Harada, Masataka Sata

161. Assessment of Second- and Third-Generation Drug-Eluting Stents on Chronic Coronary Angioscopy - Multicenter Study on Intra-Coronary AngioScopy After Stent (MICASA) Prospective Data Analysis -

Toru Miyoshi, Hiroshi Matsuoka, Hideo Kawakami, Kazuoki Dai, Tetsuya Sato, Kouki Watanabe, Masaharu Ishihara

162. Allometric Relationships for Cardiac Size and Longitudinal Function in Healthy Chinese Adults - Normal Ranges and Clinical Correlates -

Michael CH Seng, Xiayan Shen, Kangjie Wang, Daniel TT Chong, Jiang Ming Fam, Nadira Hamid, Mohammed Rizwan Amanullah, Khung Keong Yeo, See Hooi Ewe, Terrance SJ Chua, Zee Pin Ding, Anders Sahlén

163. Ultra-High-Resolution Computed Tomography Angiography for Assessment of Coronary Artery Stenosis Sadako Motoyama, Hajime Ito, Masayoshi Sarai, Yasuomi Nagahara, Keiichi Miyajima, Ryota Matsumoto, Yujiro Doi, Yumi Kataoka, Hiroshi Takahashi, Yukio Ozaki, Hiroshi Toyama, Kazuhiro Katada

164. Prognostic Value of Frailty and Diastolic Dysfunction in Elderly Patients Kenya Kusunose, Yuichiro Okushi, Hirotsugu Yamada, Susumu Nishio, Yuta Torii, Yukina Hirata, Yoshihito Saijo, Takayuki Ise, Koji Yamaguchi, Shusuke Yagi, Takeshi Soeki, Tetsuzo Wakatsuki, Masataka Sata

165. Predictive Value of Septal Flash for Reduction of Left Ventricular Systolic Function as Reflected by Global Longitudinal Strain Using Echocardiography in Patients With Isolated Complete Left Bundle-Branch Block Yonghuai Wang, Guangyuan Li, Chunyan Ma, Zhengyu Guan, Xuanyi Jin, Yang Li, Shuang Liu, Jun Yang 
166. Inter-Technique Consistency and Prognostic Value of Intra-Procedural Angiographic and Echocardiographic Assessment of Aortic Regurgitation After Transcatheter Aortic Valve Implantation

Hiroki Tateishi, Yosuke Miyazaki, Takayuki Okamura, Mohammad Abdelghani, Rodrigo Modolo, Yasuaki Wada, Shinichi Okuda, Ayumi Omuro, Toru Ariyoshi, Ayano Fujii, Tetsuro Oda, Tatsuhiro Fujimura, Takuma Nanno, Akihito Mikamo, Osama I.I. Soliman, Yoshinobu Onuma, Kimikazu Hamano, Masafumi Yano, Patrick W. Serruys

167. Optical Coherence Tomography Predictors for Recurrent Restenosis After Paclitaxel-Coated Balloon Angioplasty for Drug-Eluting Stent Restenosis

Katsuya Miura, Takeshi Tada, Seiji Habara, Akimune Kuwayama, Takenobu Shimada, Masanobu Ohya, Ryosuke Murai, Hidewo Amano, Shunsuke Kubo, Suguru Otsuru, Hiroyuki Tanaka, Yasushi Fuku, Tsuyoshi Goto, Kazushige Kadota

168. Non-Invasive Pulmonary Capillary Wedge Pressure Assessment on Speckle Tracking Echocardiography as a Predictor of New-Onset Non-Valvular Atrial Fibrillation - Four-Year Prospective Study (NIPAF Study) -

Masanori Kawasaki, Ryuhei Tanaka, Akihiro Yoshida, Maki Nagaya, Shingo Minatoguchi, Takashi Yoshizane, Takatomo Watanabe, Hiromitsu Kanamori, Koji Ono, Takeshi Hirose, Toshiyuki Noda, Sachiro Watanabe

Ischemic Heart Disease (45)

169. Efficacy and Safety of the Cholesteryl Ester Transfer Protein Inhibitor Evacetrapib in Combination With Atorvastatin in Japanese Patients With Primary Hypercholesterolemia

Tamio Teramoto, Arihiro Kiyosue, Takeshi Iimura, Yasushi Takita, Jeffrey S. Riesmeyer, Masahiro Murakami

170. Histone Deacetylase 1 Inhibition Protects Against Hypoxia-Induced Swelling in H9c2 Cardiomyocytes Through Regulating Cell Stiffness

Yi Li, Zhengyi Zhang, Xiangnan Zhou, Rui Li, Yan Cheng, Bo Shang, Yu Han, Bin Liu, Xiaodong Xie

171. Role of Routine Follow-up Coronary Angiography After Percutaneous Coronary Intervention - Systematic Review and Meta-Analysis -

Naoki Misumida, Akihiro Kobayashi, Sun Moon Kim, Ahmed Abdel-Latif, Khaled M Ziada

172. Impact of Dual Antiplatelet Therapy Beyond 1 Year on Clinical Outcomes of Patients With Stent Fracture or PeriStent Contrast Staining After Sirolimus-Eluting Stent Implantation

Yasushi Fuku, Kazushige Kadota, Hidewo Amano, Shunsuke Kubo, Suguru Otsuru, Seiji Habara, Takeshi Tada, Hiroyuki Tanaka, Tsuyoshi Goto

173. Impact of Stent Type and Presence of Vasospastic Angina on Long-Term Prognosis

Reo Hata, Naoki Oka, Shunsuke Kubo, Akimune Kuwayama, Masanobu Ohya, Takenobu Shimada, Katsuya Miura, Hidewo Amano, Yusuke Hyodo, Suguru Otsuru, Seiji Habara, Takeshi Tada, Hiroyuki Tanaka, Yasushi Fuku, Tsuyoshi Goto, Kazushige Kadota

174. First-in-Man Trial of $\mathrm{SiO}_{2}$ Inert-Coated Bare Metal Stent System in Native Coronary Stenosis - The AXETIS FIM Trial -

Taku Asano, Pannipa Suwannasom, Yuki Katagiri, Yosuke Miyazaki, Yohei Sotomi, Robin P. Kraak, Joanna Wykrzykowska, Benno J. Rensing, Jan J. Piek, Mariann Gyöngyösi, Patrick W. Serruys, Yoshinobu Onuma

175. Clinical Usefulness of Coronary Flow Reserve Ratio for the Detection of Significant Coronary Artery Disease on ${ }^{13} \mathrm{~N}-$ Ammonia Positron Emission Tomography

Naoto Kawaguchi, Hideki Okayama, Go Kawamura, Tatsuya Shigematsu, Tatsunori Takahashi, Yoshitaka Kawada, Go Hiasa, Tadakatsu Yamada, Hiroshi Matsuoka, Yukio Kazatani, Masao Miyagawa, Teruhito Mochizuki

176. Cardiovascular and Bleeding Risks in Acute Myocardial Infarction Newly Treated With Ticagrelor vs. Clopidogrel in Taiwan

Cheng-Han Lee, Ching-Lan Cheng, Yea-Huei Kao Yang, Ting-Hsing Chao, Ju-Yi Chen, Yi-Heng Li

177. Effects of Ezetimibe-Statin Combination Therapy on Coronary Atherosclerosis in Acute Coronary Syndrome Kiyoshi Hibi, Shinjo Sonoda, Masanori Kawasaki, Yutaka Otsuji, Toyoaki Murohara, Hideki Ishii, Katsuhiko Sato, Ryoji Koshida, Yukio Ozaki, Masataka Sata, Yoshihiro Morino, Tadashi Miyamoto, Tetsuya Amano, Satoshi Morita, Ken Kozuma, Kazuo Kimura, Hisayoshi Fujiwara for the Ezetimibe-ACS Investigators

178. Changes in Serum Fibroblast Growth Factor 23 in Patients With Acute Myocardial Infarction

Hideaki Takahashi, Michishige Ozeki, Tomohiro Fujisaka, Hideaki Morita, Shu-ichi Fujita, Yoshihiro Takeda, Kensaku Shibata, Koichi Sohmiya, Masaaki Hoshiga, Junko Tamaki, Nobukazu Ishizaka

179. Diastolic Dysfunction Is a Risk of Perioperative Myocardial Injury Assessed by High-Sensitivity Cardiac Troponin $\mathrm{T}$ in Elderly Patients Undergoing Non-Cardiac Surgery

Hironobu Toda, Kazufumi Nakamura, Koji Nakagawa, Atsuyuki Watanabe, Toru Miyoshi, Nobuhiro Nishii, Kazuyoshi Shimizu, Masao Hayashi, Hiroshi Morita, Hiroshi Morimatsu, Hiroshi Ito

180. Diagnosis and Management of Spontaneously Recanalized Coronary Thrombus Guided by Optical Coherence Tomography - Lessons From the French "Lotus Root” Registry -

Géraud Souteyrand, Mathieu Valladier, Nicolas Amabile, François Derimay, Brahim Harbaoui, Pierre Leddet, Pierre Barnay, Guilhem Malcles, Aurelien Mulliez, Colin Berry, Romain Eschalier, Nicolas Combaret, Pascal Motreff

181. Predictive Validity of CRUSADE, ACTION and ACUITY-HORIZONS Bleeding Risk Scores in Chinese Patients With ST-Segment Elevation Myocardial Infarction

Ran Liu, Wen Zheng, Guanqi Zhao, Xiao Wang, Xuedong Zhao, Shenghui Zhou, Shaoping Nie 
182. Two-Year Outcomes and Predictors of Target Lesion Revascularization for Non-Left Main Coronary Bifurcation Lesions Following Two-Stent Strategy With 2nd-Generation Drug-Eluting Stents

Masanobu Ohya, Takeshi Morimoto, Shunsuke Kubo, Seiji Habara, Akimune Kuwayama, Katsuya Miura, Takenobu Shimada, Hidewo Amano, Suguru Otsuru, Takeshi Tada, Hiroyuki Tanaka, Yasushi Fuku, Tsuyoshi Goto, Kazushige Kadota

183. Diagnostic Accuracy of Quantitative Flow Ratio for Assessing Myocardial Ischemia in Prior Myocardial Infarction Hiroki Emori, Takashi Kubo, Takeyoshi Kameyama, Yasushi Ino, Yoshiki Matsuo, Hironori Kitabata, Kosei Terada, Yosuke Katayama, Hiroshi Aoki, Akira Taruya, Kunihiro Shimamura, Shingo Ota, Atsushi Tanaka, Takeshi Hozumi, Takashi Akasaka

184. Intravascular Ultrasound-Derived Virtual Fractional Flow Reserve for the Assessment of Myocardial Ischemia Fumiyasu Seike, Teruyoshi Uetani, Kazuhisa Nishimura, Hiroshi Kawakami, Haruhiko Higashi, Akira Fujii, Jun Aono, Takayuki Nagai, Katsuji Inoue, Jun Suzuki, Shinji Inaba, Takafumi Okura, Kazunori Yasuda, Jitsuo Higaki, Shuntaro Ikeda

185. Microvascular Angina - Long-Term Exercise Stress Test Follow-up -

Gaetano A. Lanza, Monica Filice, Antonio De Vita, Angelo Villano, Laura Manfredonia, Priscilla Lamendola, Filippo Crea

186. Cost-Effectiveness of Statin Plus Eicosapentaenoic Acid Combination Therapy for Cardiovascular Disease Prevention in Japanese Patients With Hypercholesterolemia - An Analysis Based on the Japan Eicosapentaenoic Acid Lipid Intervention Study (JELIS) Satoshi Kodera, Hiroyuki Morita, Arihiro Kiyosue, Jiro Ando, Issei Komuro

187. Low-Dose Erythropoietin in Patients With ST-Segment Elevation Myocardial Infarction (EPO-AMI-II) - A Randomized Controlled Clinical Trial -

Tetsuo Minamino, Shuichiro Higo, Ryo Araki, Shungo Hikoso, Daisaku Nakatani, Hiroshi Suzuki, Takahisa Yamada, Masaaki Okutsu, Kouji Yamamoto, Yasushi Fujio, Yoshio Ishida, Takuya Ozawa, Kiminori Kato, Ken Toba, Yoshifusa Aizawa, Issei Komuro, EPO-AMI-II Investigators

188. Determinants and Long-Term Outcomes of Percutaneous Coronary Interventions vs. Surgery for Multivessel Disease According to Clinical Presentation

Sung-Jin Hong, Byeong-Keuk Kim, Sanghoon Shin, Yongsung Suh, Seunghwan Kim, Chul-Min Ahn, Jung-Sun Kim, Young-Guk Ko, Donghoon Choi, Myeong-Ki Hong, Yangsoo Jang

189. Protective Effects of Topiroxostat on an Ischemia-Reperfusion Model of Rat Hearts

Shogo Tanno, Kenshiro Yamamoto, Yasutaka Kurata, Maya Adachi, Yumiko Inoue, Naoyuki Otani, Mutsuo Mishima, Yasutaka Yamamoto, Masanari Kuwabara, Kazuhide Ogino, Junichiro Miake, Haruaki Ninomiya, Yasuaki Shirayoshi, Futoshi Okada, Kazuhiro Yamamoto, Ichiro Hisatome

190. Effects of Statin Intensity on Clinical Outcome in Acute Myocardial Infarction Patients

Doyeon Hwang, Hyun Kuk Kim, Joo Myung Lee, Ki Hong Choi, Jihoon Kim, Tae-Min Rhee, Jonghanne Park, Taek Kyu Park, Jeong Hoon Yang, Young Bin Song, Jin-Ho Choi, Joo-Yong Hahn, Seung-Hyuk Choi, Bon-Kwon Koo, Young Jo Kim, Shung Chull Chae, Myeong Chan Cho, Chong Jin Kim, Hyeon-Cheol Gwon, Myung Ho Jeong, Hyo-Soo Kim, The KAMIR Investigators

191. Incidence and Significance of Spontaneous ST Segment Re-elevation After Reperfused Anterior Acute Myocardial Infarction - Relationship With Infarct Size, Adverse Remodeling, and Events at 1 Year -

Léo Cuenin, Sophie Lamoureux, Mathieu Schaaf, Thomas Bochaton, Jean-Pierre Monassier, Marc J. Claeys, Gilles Rioufol, Gérard Finet, David Garcia-Dorado, Denis Angoulvant, Meyer Elbaz, Nicolas Delarche, Pierre Coste, Marc Metge, Thibault Perret, Pascal Motreff, Eric Bonnefoy-Cudraz, Gérald Vanzetto, Olivier Morel, Inesse Boussaha, Michel Ovize, Nathan Mewton

192. Rural-Urban Disparity in Emergency Care for Acute Myocardial Infarction in Japan Jun Masuda, Mikio Kishi, Naoto Kumagai, Toru Yamazaki, Kenji Sakata, Takumi Higuma, Akiyoshi Ogimoto, Kaoru Dohi, Takashi Tanigawa, Hiroyuki Hanada, Mashio Nakamura, Shigeru Sokejima, Morimasa Takayama, Jitsuo Higaki, Masakazu Yamagishi, Ken Okumura, Masaaki Ito

193. Clinical Impact of the Ankle-Brachial Index in Patients Undergoing Successful Percutaneous Coronary Intervention Seiichi Hiramori, Yoshimitsu Soga, Norihiko Kamioka, Takashi Miura, Tatsuki Doijiri, Shinichi Shirai, Kenji Ando

194. Survey on the Status of Smoking Inside Eating Establishments in the Cities of Kobe and Amagasaki Yukihito Sato, Yoshio Kita, Masatoshi Shimada, Kiyomi Matsushita, Hisayoshi Fujiwara

Absolute Myocardial Blood Flow After Elective Percutaneous Coronary Intervention Evaluated on Phase-Contrast Cine Cardiovascular Magnetic Resonance Imaging

Rikuta Hamaya, Akinori Sugano, Yoshihisa Kanaji, Tadashi Fukuda, Yoshinori Kanno, Taishi Yonetsu, Eisuke Usui, Masahiro Hoshino, Masahiro Hada, Hiroaki Ohya, Yohei Sumino, Haruhito Yuki, Tadashi Murai, Tetsumin Lee, Tsunekazu Kakuta

196. Comparison of Clinical Outcomes Between Ticagrelor and Prasugrel in Patients With ST-Segment Elevation Myocardial Infarction - Results From the Korea Acute Myocardial Infarction Registry-National Institutes of Health -

Min Chul Kim, Myung Ho Jeong, Doo Sun Sim, Young Joon Hong, Ju Han Kim, Youngkeun Ahn, Tae Hoon Ahn, Ki Bae Seung, Dong-Ju Choi, Hyo-Soo Kim, Hyeon Cheol Gwon, In Whan Seong, Kyoung-Kook Hwang, Shung Chull Chae, Seung Ho Hur, Kwang Soo Cha, Seok Kyu Oh, Jei Keon Chae, Korea Acute Myocardial Infarction-National Institutes of Health Registry Investigators 
197. In-Hospital Bleeding and Utility of a Maintenance Dose of Prasugrel $2.5 \mathrm{mg}$ in High Bleeding Risk Patients With Acute Coronary Syndrome

Masanobu Ohya, Takenobu Shimada, Kohei Osakada, Akimune Kuwayama, Katsuya Miura, Ryosuke Murai, Hidewo Amano, Shunsuke Kubo, Suguru Otsuru, Seiji Habara, Takeshi Tada, Hiroyuki Tanaka, Yasushi Fuku, Harumi Katoh, Tsuyoshi Goto, Kazushige Kadota

198. CAMI-NSTEMI Score - China Acute Myocardial Infarction Registry-Derived Novel Tool to Predict In-Hospital Death in Non-ST Segment Elevation Myocardial Infarction Patients -

Rui Fu, Chenxi Song, Jingang Yang, Yan Wang, Bao Li, Haiyan Xu, Xiaojin Gao, Wei Li, Jia Liu, Kefei Dou, Yuejin Yang on behalf of the CAMI Registry Study Group

199. Effect of Sitagliptin on Coronary Flow Reserve Assessed by Magnetic Resonance Imaging in Type 2 Diabetic Patients With Coronary Artery Disease

Keishi Moriwaki, Tetsushiro Takeuchi, Naoki Fujimoto, Toshiki Sawai, Yuichi Sato, Naoto Kumagai, Jun Masuda, Shiro Nakamori, Masaki Ishida, Norikazu Yamada, Mashio Nakamura, Hajime Sakuma, Masaaki Ito, Kaoru Dohi

200. P-Selectin Glycoprotein Ligand-1 (PSGL-1) Expressing CD4 T Cells Contribute Plaque Instability in Acute Coronary Syndrome

Kazutaka Kitamura, Kayoko Sato, Motoji Sawabe, Masayuki Yoshida, Nobuhisa Hagiwara

201. Revascularization vs. Medical Therapy for Coronary Chronic Total Occlusions in Patients With Chronic Kidney Disease

Chung Hun Kim, Jeong Hoon Yang, Taek Kyu Park, Young Bin Song, Joo-Yong Hahn, Jin-Ho Choi, Sang Hoon Lee, Hyeon-Cheol Gwon, Joonghyun Ahn, Keumhee Chough Carriere, Seung-Hyuk Choi

202. Efficacy of 2.5-mg Prasugrel in Elderly or Low-Body-Weight Patients Shinichi Wakabayashi, Noritaka Ariyoshi, Hideki Kitahara, Kenichi Fujii, Yoshihide Fujimoto, Yoshio Kobayashi

203. Transplantation of Endothelial Progenitor Cells Overexpressing miR-126-3p Improves Heart Function in Ischemic Cardiomyopathy

Hong Li, Qiang Liu, Ningfu Wang, Yizhou Xu, Lan Kang, Yaqi Ren, Gangjie Zhu

204. Impact of the Coronary Artery Calcium Score on Mid- to Long-Term Cardiovascular Mortality and Morbidity Measured With Coronary Computed Tomography Angiography

Hideya Yamamoto, Toshiro Kitagawa, Eiji Kunita, Hiroto Utsunomiya, Atsuhiro Senoo, Yumiko Nakamoto, Yasuki Kihara

205. Alamandine Protects the Heart Against Reperfusion Injury via the MrgD Receptor Byung Mun Park, Hoang Thi Ai Phuong, Lamei Yu, Suhn Hee Kim

206. Early-Phase Vascular Healing of Bioabsorbable vs. Durable Polymer-Coated Everolimus-Eluting Stents in Patients With ST-Elevation Myocardial Infarction - 2-Week and 4-Month Analyses With Optical Coherence Tomography Masahiro Shimoda, Hirohiko Ando, Kazuhiro Naito, Akihiro Suzuki, Shinichiro Sakurai, Yusuke Nakano, Akiyoshi Kurita, Katsuhisa Waseda, Hiroaki Takashima, Kenta Murotani, Tadayuki Uetani, Tetsuya Amano

207. Cost-Effectiveness of PCSK9 Inhibitor Plus Statin in Patients With Triple-Vessel Coronary Artery Disease in Japan Satoshi Kodera, Hiroyuki Morita, Arihiro Kiyosue, Jiro Ando, Tomoyuki Takura, Issei Komuro

Stimulation of the Beta2 Adrenergic Receptor at Reperfusion Limits Myocardial Reperfusion Injury via an Interleukin-10-Dependent Anti-Inflammatory Pathway in the Spleen

Yikui Tian, Bin Miao, Eric J. Charles, Di Wu, Irving L. Kron, Brent A. French, Zequan Yang

209. Diagnostic Performance of the Simultaneous Acquisition Rest $99 \mathrm{~m}$ Tc-Tetrofosmin/Stress ${ }^{201}$ Tl Dual-Isotope Protocol With a Semiconductor Camera - Comparison With the Rest-Stress 99mTc-Tetrofosmin Protocol Itta Kawamura, Ryo Kajiura, Yusuke Motoji, Syuichi Okamoto, Toru Tanigaki, Hiroyuki Omori, Tetsuo Hirata, Jun Kikuchi, Hideaki Ota, Yoshihiro Sobue, Taiji Miyake, Tomohiro Tsunekawa, Takayoshi Kato, Yoshiaki Kawase, Munenori Okubo, Hiroki Kamiya, Kunihiko Tsuchiya, Shinji Tomita, Akihiro Hirakawa, Takeshi Kondo, Takahiko Suzuki, Hitoshi Matsuo

210. Number of Board-Certified Cardiologists and Acute Myocardial Infarction-Related Mortality in Japan - JROAD and JROAD-DPC Registry Analysis -

Koshiro Kanaoka, Satoshi Okayama, Kihei Yoneyama, Michikazu Nakai, Kunihiro Nishimura, Hiroyuki Kawata, Manabu Horii, Rika Kawakami, Hiroyuki Okura, Yoshihiro Miyamoto, Yoshihiro Akashi, Yoshihiko Saito

211. Significant Association Between Coronary Artery Low-Attenuation Plaque Volume and Apnea-Hypopnea Index, But Not Muscle Sympathetic Nerve Activity, in Patients With Obstructive Sleep Apnea Syndrome

Takuto Hamaoka, Hisayoshi Murai, Shuichi Kaneko, Soichiro Usui, Oto Inoue, Hiroyuki Sugimoto, Yusuke Mukai, Yoshitaka Okabe, Hideki Tokuhisa, Shinichiro Takashima, Takeshi Kato, Hiroshi Furusho, Soichiro Kashiwaya, Yu Sugiyama, Yasuto Nakatsumi, Shigeo Takata, Masayuki Takamura

212. Clinical Predictors of Recurrent Ventricular Arrhythmias in Secondary Prevention Implantable Cardioverter Defibrillator Recipients With Coronary Artery Disease - Lower Left Ventricular Ejection Fraction and Incomplete Revascularization -

Toshiki Takano, Komei Tanaka, Kazuyuki Ozaki, Akinori Sato, Kenichi Iijima, Takao Yanagawa, Daisuke Izumi, Takuya Ozawa, Koichi Fuse, Masahito Sato, Naohito Tanabe, Tohru Minamino 
213. Retrospective Comparison of Long-Term Clinical Outcomes Between Percutaneous Coronary Intervention and Medical Therapy in Stable Coronary Artery Disease With Gray Zone Fractional Flow Reserve - COMFORTABLE Retrospective Study -

Takashi Kubo, Masahiro Takahata, Kosei Terada, Kazuya Mori, Yu Arita, Yasushi Ino, Yoshiki Matsuo, Hironori Kitabata, Yasutsugu Shiono, Kunihiro Shimamura, Takeyoshi Kameyama, Hiroki Emori, Yosuke Katayama, Takashi Tanimoto, Takashi Akasaka

Metabolic Disorder (9)

214. Clinical Features and Gaps in the Management of Probable Familial Hypercholesterolemia and Cardiovascular Disease

Barak Zafrir, Ayman Jubran, Gil Lavie, David A. Halon, Moshe Y. Flugelman, Chen Shapira

215. Renin-Angiotensin System Inhibitors Can Prevent Intravenous Lipid Infusion-Induced Myocardial Microvascular Dysfunction and Leukocyte Activation

Takanori Yasu, Akiko Mutoh, Hiroshi Wada, Mayumi Kobayashi, Yuji Kikuchi, Shinichi Momomura, Shinichiro Ueda

216. Association of Epicardial, Visceral, and Subcutaneous Fat With Cardiometabolic Diseases Fumi Sato, Norikazu Maeda, Takayuki Yamada, Hideyuki Namazui, Shiro Fukuda, Tomoaki Natsukawa, Hirofumi Nagao, Jun Murai, Shigeki Masuda, Yoshimitsu Tanaka, Yoshinari Obata, Yuya Fujishima, Hitoshi Nishizawa, Tohru Funahashi, Iichiro Shimomura

217. Circulating Fatty Acid-Binding Protein 4 Concentration Predicts the Progression of Carotid Atherosclerosis in a General Population Without Medication

Masato Furuhashi, Satoshi Yuda, Atsuko Muranaka, Mina Kawamukai, Megumi Matsumoto, Marenao Tanaka, Norihito Moniwa, Hirofumi Ohnishi, Shigeyuki Saitoh, Kazuaki Shimamoto, Tetsuji Miura

218. Effect of Rosuvastatin on Cholesterol Efflux Capacity and Endothelial Function in Type 2 Diabetes Mellitus and Dyslipidemia

Kyong Yeun Jung, Kyoung Min Kim, Sun Kyoung Han, Han Mi Yun, Tae Jung Oh, Sung Hee Choi, Kyong Soo Park, Hak Chul Jang, Soo Lim

219. Plasma Xanthine Oxidoreductase Activity as a Novel Biomarker of Metabolic Disorders in a General Population Masato Furuhashi, Megumi Matsumoto, Marenao Tanaka, Norihito Moniwa, Takayo Murase, Takashi Nakamura, Hirofumi Ohnishi, Shigeyuki Saitoh, Kazuaki Shimamoto, Tetsuji Miura

220. MicroRNA-377 Inhibits Atherosclerosis by Regulating Triglyceride Metabolism Through the DNA Methyltransferase 1 in Apolipoprotein E-Knockout Mice Ling-Yan Chen, Xiao-Dan Xia, Zhen-Wang Zhao, Duo Gong, Xiao-Feng Ma, Xiao-Hua Yu, Qiang Zhang, Si-Qi Wang, Xiao-Yan Dai, Xi-Long Zheng, Da-Wei Zhang, Wei-Dong Yin, Chao-Ke Tang

221. Association of Serum Triglycerides With Arterial Stiffness in Subjects With Low Levels of Low-Density Lipoprotein Cholesterol

Shin Kawasoe, Kazuki Ide, Tomoko Usui, Takuro Kubozono, Shiro Yoshifuku, Hironori Miyahara, Shigeho Maenohara, Mitsuru Ohishi, Koji Kawakami

222. Correlations Between Serum Cholesterol and Vascular Lesions in Fabry Disease Patients Hiroki Katsuta, Kazuya Tsuboi, Hiroshi Yamamoto, Hiromi Goto

Molecular Cardiology (3)

223. Association Between a CCL17 Genetic Variant and Risk of Coronary Artery Disease in a Chinese Han Population Yicong Ye, Xinglin Yang, Bo Long, Haiyu Pang, Yicheng Zhu, Shuyang Zhang

224. Endogenous Interleukin- $1 \beta$ Is Implicated in Intraplaque Hemorrhage in Apolipoprotein E Gene Null Mice Etsu Suzuki, Hiroyuki Imuta, Daishi Fujita, Masao Takahashi, Shigeyoshi Oba, Arihiro Kiyosue, Hiroaki Nishimatsu

225. Apolipoprotein A-1 Binding Protein Inhibits Inflammatory Signaling Pathways by Binding to Apolipoprotein A-1 in THP-1 Macrophages

Min Zhang, Guo-Jun Zhao, Kai Yin, Xiao-Dan Xia, Duo Gong, Zhen-Wang Zhao, Ling-Yan Chen, Xi-Long Zheng, Xiao-Er Tang, Chao-Ke Tang

Myocardial Disease (5)

226. Third and Fourth Heart Sounds and Myocardial Fibrosis in Hypertrophic Cardiomyopathy Yoshimi Sato, Tatsuya Kawasaki, Sakiko Honda, Kuniyasu Harimoto, Shigeyuki Miki, Tadaaki Kamitani, Hirokazu Shiraishi, Satoaki Matoba

227. Patients' Characteristics and Clinical Course of Hypertrophic Cardiomyopathy in a Regional Japanese Cohort - Results From Kochi RYOMA Study -

Toru Kubo, Takayoshi Hirota, Yuichi Baba, Yuri Ochi, Asa Takahashi, Naohito Yamasaki, Naohisa Hamashige, Katsuhito Yamamoto, Fumiaki Kondo, Kanji Bando, Eisuke Yamada, Takashi Furuno, Toshikazu Yabe, Yoshinori L Doi, Hiroaki Kitaoka

228. Late Gadolinium Enhancement for Prediction of Mutation-Positive Hypertrophic Cardiomyopathy on the Basis of Panel-Wide Sequencing Ryota Teramoto, Noboru Fujino, Tetsuo Konno, Akihiro Nomura, Yoji Nagata, Toyonobu Tsuda, Hayato Tada, Kenji Sakata, Masakazu Yamagishi, Kenshi Hayashi, Masa-aki Kawashiri 
229. Clinical Correlates and Prognostic Value of Elevated Right Atrial Pressure in Patients With Hypertrophic Cardiomyopathy

Stephen A. McCullough, Michael A. Fifer, Pouya Mohajer, Patricia A. Lowry, Caitlin O'Callaghan Reen, Aaron L. Baggish, Gus J. Vlahakes, Yuichi J. Shimada

230. Clinical and Echocardiographic Impact of Tafazzin Variants on Dilated Cardiomyopathy Phenotype in Left Ventricular Non-Compaction Patients in Early Infancy

Keiichi Hirono, Yukiko Hata, Makoto Nakazawa, Nobuo Momoi, Tohru Tsuji, Taro Matsuoka, Mamoru Ayusawa, Yuriko Abe, Tamaki Hayashi, Nobuyuki Tsujii, Tadaaki Abe, Heima Sakaguchi, Ce Wang, Asami Takasaki, Shinya Takarada, Mako Okabe, Nariaki Miyao, Hideyuki Nakaoka, Keijiro Ibuki, Kazuyoshi Saito, Sayaka Ozawa, Naoki Nishida, Neil E. Bowles, Fukiko Ichida

Pediatric Cardiology and Adult Congenital Heart Disease (14)

231. Familial Aggregation and Heritability of Congenital Heart Defects Chang-Fu Kuo, Yu-Sheng Lin, Shang-Hung Chang, I-Jun Chou, Shue-Fen Luo, Lai-Chu See, Kuang-Hui Yu, Lu-Shuang Huang, Pao-Hsien Chu

232. Nationwide Survey of Patients With Giant Coronary Aneurysm Secondary to Kawasaki Disease 1999-2010 in Japan Ryuji Fukazawa, Tohru Kobayashi, Masashi Mikami, Tsutomu Saji, Kenji Hamaoka, Hitoshi Kato, Hiroyuki Suzuki, Etsuko Tsuda, Mamoru Ayusawa, Masaru Miura, Ryota Ebata, Tomio Kobayashi, Mayumi Yashiro, Shunichi Ogawa

233. On What Day of Illness Does the Dilatation of Coronary Arteries in Patients With Kawasaki Disease Begin? Shigeto Fuse, Toshihiko Mori, Yuki Kuroiwa, Satoshi Hirakawa

234. Cognitive Development After Kawasaki Disease - Clinical Study and Validation Using a Nationwide PopulationBased Cohort Liang-Jen Wang, Ho-Chang Kuo

235. Standard Values and Characteristics of Electrocardiographic Findings in Children and Adolescents Masao Yoshinaga, Mari Iwamoto, Hitoshi Horigome, Naokata Sumitomo, Hiroya Ushinohama, Naomi Izumida, Nobuo Tauchi, Tatsuya Yoneyama, Katsumi Abe, Masami Nagashima

236. Clinical Characteristics of Adult Patients With Congenital Heart Disease Hospitalized for Acute Heart Failure Jun Negishi, Hideo Ohuchi, Aya Miyazaki, Etsuko Tsuda, Isao Shiraishi, Kenichi Kurosaki

237. Clinical Predictors of Right Ventricular Myocardial Fibrosis in Patients With Repaired Tetralogy of Fallot Takashi Kido, Takayoshi Ueno, Masaki Taira, Hideto Ozawa, Koichi Toda, Toru Kuratani, Yoshiki Sawa

238. Liver Cirrhosis and/or Hepatocellular Carcinoma Occurring Late After the Fontan Procedure - A Nationwide Survey in Japan -

Masanari Kuwabara, Koichiro Niwa, Tomohiko Toyoda, Takeaki Shirai, Shigeru Tateno, Hideo Ohuchi, Yasuhiko Tanaka, Fukiko Ichida, Tomoo Fujisawa, Teiji Akagi, Yoshiki Mori for the Research Committee of the Japanese Society of Pediatric Cardiology and Cardiac Surgery

239. Is Trisomy 21 a Risk Factor for Rapid Progression of Pulmonary Arteriopathy? - Revisiting Histopathological Characteristics Using 282 Lung Biopsy Specimens -

Naoki Masaki, Yuriko Saiki, Masato Endo, Kay Maeda, Osamu Adachi, Masatoshi Akiyama, Shunsuke Kawamoto, Yoshikatsu Saiki

240. Prediction of Fontan-Associated Liver Disease Using a Novel Cine Magnetic Resonance Imaging "Vortex Flow Map" in the Right Atrium

Umiko Ishizaki, Michinobu Nagao, Yumi Shiina, Kenji Fukushima, Tatsunori Takahashi, Yamato Shimomiya, Yuka Matsuo, Kei Inai, Shuji Sakai

241. Autonomic Function and QT Interval During Night-Time Sleep in Infant Long QT Syndrome Masao Yoshinaga, Yu Kucho, Hiroya Ushinohama, Yuichi Ishikawa, Seiko Ohno, Hiromitsu Ogata

242. Amniotic Fluid Natriuretic Peptide Levels in Fetuses With Congenital Heart Defects or Arrhythmias Takekazu Miyoshi, Hiroshi Hosoda, Takashi Umekawa, Takashi Asada, Akihiro Fujiwara, Ken-ichi Kurosaki, Isao Shiraishi, Michikazu Nakai, Kunihiro Nishimura, Mikiya Miyazato, Kenji Kangawa, Tomoaki Ikeda, Jun Yoshimatsu, Naoto Minamino

243. Tentative Screening Criteria for Short QT Interval in Children and Adolescents Daisuke Hazeki, Yumiko Ninomiya, Kentaro Ueno, Masao Yoshinaga

244. One-Year Follow-up After Tetralogy of Fallot Total Repair Preserving Pulmonary Valve and Avoiding Right Ventriculotomy

Jae Gun Kwak, Woong-Han Kim, Eung Re Kim, Jae Hong Lim, Jooncheol Min

Peripheral Vascular Disease (17)

245. Barthel Index and Outcome of Open Bypass for Critical Limb Ischemia Shinsuke Mii, Atsushi Guntani, Eisuke Kawakubo, Hirofumi Shimazoe

246. Pathological Quantification of Carotid Artery Plaque Instability in Patients Undergoing Carotid Endarterectomy Takao Konishi, Naohiro Funayama, Tadashi Yamamoto, Tohru Morita, Daisuke Hotta, Ryota Nomura, Yusuke Nakagaki, Takeo Murahashi, Kenji Kamiyama, Tetsuyuki Yoshimoto, Takeshi Aoki, Hiroshi Nishihara, Shinya Tanaka 
247. Association Between Preoperative Frailty and Mortality in Patients With Critical Limb Ischemia Following Infrainguinal Bypass Surgery - Usefulness of the Barthel Index -

Akio Kodama, Akio Koyama, Masayuki Sugimoto, Kiyoaki Niimi, Hiroshi Banno, Kimihiro Komori

248. Edoxaban Enhances Thromboprophylaxis by Physiotherapy After Total Knee Arthroplasty - The Randomized Controlled ESCORT-TKA Trial -

Daisuke Sueta, Koichi Kaikita, Nobukazu Okamoto, Soichiro Yamabe, Masanobu Ishii, Yuichiro Arima, Miwa Ito, Yu Oimatsu, Tatsuro Mitsuse, Satomi Iwashita, Eiichi Nakamura, Seiji Hokimoto, Hiroshi Mizuta, Hisao Ogawa, Kenichi Tsujita for the ESCORT-TKA Study Investigators

249. Impact of Objective Malnutrition Status on the Clinical Outcomes in Patients With Peripheral Artery Disease Following Endovascular Therapy

Miyuki Yokoyama, Tetsu Watanabe, Yoichiro Otaki, Ken Watanabe, Taku Toshima, Takayuki Sugai, Tetsuya Takahashi, Daisuke Kinoshita, Harutoshi Tamura, Satoshi Nishiyama, Hiroki Takahashi, Takanori Arimoto, Tetsuro Shishido, Sou Yamauchi, Tamon Yamanaka, Takuya Miyamoto, Isao Kubota

250. Transcutaneous Exercise Oximetry for Patients With Claudication - A Retrospective Review of Approximately 5,000 Consecutive Tests Over 15 Years -

Pierre Abraham, Christophe Colas-Ribas, Isabelle Signolet, Myriam Ammi, Mathieu Feuilloy, Jean Picquet, Samir Henni

251. Long-Term Clinical Outcomes Survey of Bone Marrow-Derived Cell Therapy in Critical Limb Ischemia in Japan Kazuhisa Kondo, Kenji Yanishi, Ryo Hayashida, Satoshi Shintani, Rei Shibata, Kenta Murotani, Masahiko Ando, Masaaki Mizuno, Tadami Fujiwara, Toyoaki Murohara, Satoaki Matoba, TACT Follow-up Study Investigators

252. Impact of Stent-to-Vessel Diameter Ratio on Restenosis in the Superficial Femoral Artery After Endovascular Therapy Naoya Kurata, Osamu Iida, Tatsuya Shiraki, Masashi Fujita, Masaharu Masuda, Shin Okamoto, Takayuki Ishihara, Kiyonori Nanto, Takashi Kanda, Akihiro Sunaga, Takuya Tsujimura, Mitsuyoshi Takahara, Toshiaki Mano

253. Clinical Outcomes of Subintimal vs. Intraluminal Revascularization Approaches for Long Femoropopliteal Occlusions in a Korean Multicenter Retrospective Registry Cohort

Kyu Kim, Young-Guk Ko, Chul-Min Ahn, Pil-Ki Min, Jae-Hwan Lee, Chang-Hwan Yoon, Cheol Woong Yu, Seung Whan Lee, Sang-Rok Lee, Seung Hyuk Choi, Yoon Seok Koh, In-Ho Chae, Donghoon Choi

254. Drug-Coated Balloon vs. Conventional Balloon Angioplasty in Dialysis Patients With Symptomatic Femoropopliteal Disease - A Matched Comparison -

Hsin-Hua Chou, Hsuan-Li Huang, Chien-An Hsieh, Shih-Jung Jang, I-Shiang Tzeng, Yu-Lin Ko

255. Cardiovascular Outcome and Mortality in Patients Undergoing Endovascular Treatment for Symptomatic Peripheral Artery Disease - Short-Term Results of the Toma-Code Registry -

Michiaki Higashitani, Yukari Uemura, Atsushi Mizuno, Makoto Utsunomiya, Tetsuo Yamaguchi, Akihiro Matsui, Shunsuke Ozaki, Kazuki Tobita, Atsushi Tosaka, Akitsugu Oida, Kenji Suzuki, Takahide Kodama, Kentaro Jujo, Tatsuki Doijiri, Yasuhiro Takahashi, Shunsuke Matsuno, Nobuhito Kaneko, Akira Moriguchi, Shohei Kishi, Hitoshi Anzai on behalf of Toma-Code Registry Investigators

256. Circulating Surfactant Protein-D Is Associated With Clinical Outcomes in Peripheral Artery Disease Patients Following Endovascular Therapy

Yoichiro Otaki, Tetsu Watanabe, Hiroki Takahashi, Takayuki Sugai, Miyuki Yokoyama, Satoshi Nishiyama, Takanori Arimoto, Tetsuro Shishido, Takuya Miyamoto, Tamon Yamanaka, Isao Kubota, Masafumi Watanabe

257. Hybrid Viabahn-Assisted Bypass for Long Femoro-Popliteal Occlusive Disease - Midterm Results Po-Lin Chen, Ting-Chao Lin, I-Ming Chen

Outcome From a Randomized Controlled Clinical Trial - Improvement of Peripheral Arterial Disease by Granulocyte Colony-Stimulating Factor-Mobilized Autologous Peripheral-Blood-Mononuclear Cell Transplantation (IMPACT) -

Takashi Horie, Seiji Yamazaki, Sayaka Hanada, Shuzo Kobayashi, Tatsuo Tsukamoto, Tetsuya Haruna, Katsuhiko Sakaguchi, Ken Sakai, Hideaki Obara, Kiyofumi Morishita, Kenichi Saigo, Yoshiaki Shintani, Kohmei Kubo, Junichi Hoshino, Teiji Oda, Eiji Kaneko, Masaharu Nishikido, Tetsuya Ioji, Hideaki Kaneda, Masanori Fukushima for the Japan Study Group of Peripheral Vascular Regeneration Cell Therapy (JPRCT)

259. Length and Cost of Hospital Stay in Poor-Risk Patients With Critical Limb Ischemia Undergoing Revascularization Mitsuyoshi Takahara, Osamu Iida, Yoshimitsu Soga, Nobuyoshi Azuma, Shinsuke Nanto on behalf of the PRIORITY Investigators

260. Beneficial Effect of Endovascular Treatment on Villalta Score in Japanese Patients With Chronic Iliofemoral Venous Thrombosis and Post-Thrombotic Syndrome

Jin Ueda, Akihiro Tsuji, Takeshi Ogo, Ryotaro Asano, Nao Konagai, Shigefumi Fukui, Yoshiaki Morita, Tetsuya Fukuda, Satoshi Yasuda

ecreased Psoas Muscle Computed Tomography Value Predicts Poor Outcome in Peripheral Artery Disease Takayuki Sugai, Tetsu Watanabe, Yoichiro Otaki, Jun Goto, Ken Watanabe, Taku Toshima, Tetsuya Takahashi, Miyuki Yokoyama, Harutoshi Tamura, Satoshi Nishiyama, Takanori Arimoto, Hiroki Takahashi, Tetsuro Shishido, Masafumi Watanabe 
Preventive Medicine (3)

262. Effect of Intensive and Standard Pitavastatin Treatment With or Without Eicosapentaenoic Acid on Progression of Coronary Artery Calcification Over 12 Months - Prospective Multicenter Study -

Toru Miyoshi, Kunihisa Kohno, Hirohiko Asonuma, Satoru Sakuragi, Makoto Nakahama, Yusuke Kawai, Tadahisa Uesugi, Takefumi Oka, Mitsuru Munemasa, Natsuki Takahashi, Naoki Mukohara, Seiji Habara, Yasushi Koyama, Kazufumi Nakamura, Hiroshi Ito on behalf of the PEACH Investigators

263. Effect of Smoking Status on Monocyte Tissue Factor Activity, Carotid Atherosclerosis and Long-Term Prognosis in Metabolic Syndrome

Keiichi Kohashi, Akihiro Nakagomi, Taichirou Morisawa, Ikuko Endoh, Naomi Kawaguchi, Yoshiki Kusama, Wataru Shimizu

264. Excessive Daytime Sleepiness Is Associated With Depression Scores, But Not With Sleep-Disordered Breathing in Patients With Cardiovascular Diseases

Mikiko Ohashi, Takashi Kohno, Shun Kohsaka, Ryoma Fukuoka, Kentaro Hayashida, Shinsuke Yuasa, Motoaki Sano, Seiji Takatsuki, Keiichi Fukuda

Pulmonary Circulation (9)

265. Effectiveness and Outcome of Pulmonary Arterial Hypertension-Specific Therapy in Japanese Patients With Pulmonary Arterial Hypertension

Yuichi Tamura, Hiraku Kumamaru, Toru Satoh, Hiroaki Miyata, Aiko Ogawa, Nobuhiro Tanabe, Masaru Hatano, Atsushi Yao, Kohtaro Abe, Ichizo Tsujino, Keiichi Fukuda, Hiroshi Kimura, Masataka Kuwana, Hiromi Matsubara, Koichiro Tatsumi on behalf of the Japan PH Registry (JAPHR) Network

266. Clinical Impact of Main Pulmonary Artery Dilatation on Outcome in Pediatric Idiopathic and Heritable Pulmonary Arterial Hypertension

Satoshi Ikehara, Shinichi Takatsuki, Tomotaka Nakayama, Kazuyuki Naoi, Hiroyuki Matsuura, Tsutomu Saji

267. Predictors of Favorable Responses to Immunosuppressaive Treatment in Pulmonary Arterial Hypertension Associated With Connective Tissue Disease

Hidekata Yasuoka, Yuichiro Shirai, Yuichi Tamura, Tsutomu Takeuchi, Masataka Kuwana

268. Incidence, Characteristics and Management of Venous Thromboembolism in Japan During 2011

Satoshi Ota, Akimasa Matsuda, Yoshito Ogihara, Norikazu Yamada, Mashio Nakamura, Takuya Mori, Masayuki Hamada, Takao Kobayashi, Masaaki Ito

269. Tricuspid Regurgitation Peak Gradient (TRPG)/Tricuspid Annulus Plane Systolic Excursion (TAPSE) - A Novel Parameter for Stepwise Echocardiographic Risk Stratification in Normotensive Patients With Acute Pulmonary Embolism -

Michał Ciurzyński, Katarzyna Kurnicka, Barbara Lichodziejewska, Marta Kozłowska, Magdalena Pływaczewska, Piotr Sobieraj, Olga Dzikowska-Diduch, Sylwia Goliszek, Piotr Bienias, Maciej Kostrubiec, Piotr Pruszczyk

70. Long-Term Outcome of Chronic Thromboembolic Pulmonary Hypertension at a Single Japanese Pulmonary Endarterectomy Center

Hideki Miwa, Nobuhiro Tanabe, Takayuki Jujo, Fumiaki Kato, Rie Anazawa, Keiko Yamamoto, Akira Naito, Hajime Kasai, Rintaro Nishimura, Rika Suda, Toshihiko Sugiura, Seiichiro Sakao, Keiichi Ishida, Masahisa Masuda, Koichiro Tatsumi

271. Outcomes of Pulmonary Embolectomy for Acute Pulmonary Embolism

Masahito Minakawa, Ikuo Fukuda, Hiroaki Miyata, Noboru Motomura, Shinichi Takamoto, Satoshi Taniguchi, Kazuyuki Daitoku, Norihiro Kondo, Japan Cardiovascular Surgery Database Organization

272. Pulmonary Artery Wall Thickness Assessed by Optical Coherence Tomography Correlates With Pulmonary Hemodynamics in Children With Congenital Heart Disease Yukako Homma, Yasunobu Hayabuchi, Akemi Ono, Shoji Kagami

273. Nocturnal Hypoxemia, But Not Sleep Apnea, Is Associated With a Poor Prognosis in Patients With Pulmonary Arterial Hypertension

Mika Nagaoka, Ayumi Goda, Kaori Takeuchi, Hanako Kikuchi, Mayumi Finger, Takumi Inami, Kyoko Soejima, Toru Satoh

Regenerative Medicine (2)

274. Mobilized Muse Cells After Acute Myocardial Infarction Predict Cardiac Function and Remodeling in the Chronic Phase

Toshiki Tanaka, Kazuhiko Nishigaki, Shingo Minatoguchi, Takahide Nawa, Yoshihisa Yamada, Hiromitsu Kanamori, Atsushi Mikami, Hiroaki Ushikoshi, Masanori Kawasaki, Mari Dezawa, Shinya Minatoguchi

275. Therapeutic Efficacy of Autologous Non-Mobilized Enriched Circulating Endothelial Progenitors in Patients With Critical Limb Ischemia - The SCELTA Trial -

Francesco Liotta, Francesco Annunziato, Sergio Castellani, Maria Boddi, Brunetto Alterini, Giovanni Castellini, Benedetta Mazzanti, Lorenzo Cosmi, Manlio Acquafresca, Filippo Bartalesi, Beatrice Dilaghi, Walter Dorigo, Gabriele Graziani, Benedetta Bartolozzi, Guido Bellandi, Giulia Carli, Alessandro Bartoloni, Aaron Fargion, Filippo Fassio, Paolo Fontanari, Giancarlo Landini, Eleonora A.M. Lucente, Stefano Michelagnoli, Carolina Orsi Battaglini, Grazia Panigada, Clara Pigozzi, Valentina Querci, Veronica Santarlasci, Paola Parronchi, Nicola Troisi, Cristiana Baggiore, Paola Romagnani, Edoardo Mannucci, Riccardo Saccardi, Carlo Pratesi, Gianfranco Gensini, Sergio Romagnani, Enrico Maggi 
Renal Disease (1)

276. Association Between Elevated Echocardiographic Index of Left Ventricular Filling Pressure and Acute Kidney Injury After Off-Pump Coronary Artery Surgery

Min Hur, Karam Nam, Woo Young Jo, Gahyun Kim, Won Ho Kim, Jae-Hyon Bahk

Stroke (8)

277. Prognostic Significance of Blood Urea Nitrogen in Acute Ischemic Stroke

Shoujiang You, Danni Zheng, Chongke Zhong, Xianhui Wang, Weiting Tang, Liqin Sheng, Cheng Zheng, Yongjun Cao, Chun-Feng Liu

278. Fibrin Clot Architecture in Acute Ischemic Stroke Treated With Mechanical Thrombectomy With Stent-Retrievers - Cohort Study -

Dagmar Krajičková, Antonín Krajina, Ivo Šteiner, Oldřich Vyšata, Roman Herzig, Miroslav Lojík, Vendelín Chovanec, Jan Raupach, Ondřej Renc, Jan Waishaupt, Eva Vítková, Petr Dulíček, Pavla Čabelková, Martin Vališ

279. Insufficient Warfarin Therapy Is Associated With Higher Severity of Stroke Than No Anticoagulation in Patients With Atrial Fibrillation and Acute Anterior-Circulation Stroke

Yuki Sakamoto, Seiji Okubo, Chikako Nito, Satoshi Suda, Noriko Matsumoto, Yasuhiro Nishiyama, Junya Aoki, Takashi Shimoyama, Takuya Kanamaru, Kentaro Suzuki, Masahiro Mishina, Kazumi Kimura

280. Seasonal Variations in Neurological Severity and Outcomes of Ischemic Stroke - 5-Year Single-Center Observational Study -

Kazunori Toyoda, Masatoshi Koga, Hiroshi Yamagami, Chiaki Yokota, Shoichiro Sato, Manabu Inoue, Tomotaka Tanaka, Kaoru Endo, Jun Fujinami, Masafumi Ihara, Kazuyuki Nagatsuka, Kazuo Minematsu

281. High Cardiac Troponin I Is Associated With Transesophageal Echocardiographic Risk of Thromboembolism and Ischemic Stroke Events in Non-Valvular Atrial Fibrillation Patients

Shuhei Tanaka, Tadakazu Hirai, Kyoko Inao, Nobuyuki Fukuda, Keiko Nakagawa, Hiroshi Inoue, Koichiro Kinugawa

282. Two-Year Outcomes of Anticoagulation for Acute Ischemic Stroke With Nonvalvular Atrial Fibrillation - SAMURAI-NVAF Study -

Sohei Yoshimura, Masatoshi Koga, Shoichiro Sato, Kenichi Todo, Hiroshi Yamagami, Masaya Kumamoto, Ryo Itabashi, Tadashi Terasaki, Kazumi Kimura, Yoshiki Yagita, Yoshiaki Shiokawa, Kenji Kamiyama, Satoshi Okuda, Yasushi Okada, Shunya Takizawa, Yasuhiro Hasegawa, Tomoaki Kameda, Satoshi Shibuya, Yoshinari Nagakane, Yasuhiro Ito, Hideki Matsuoka, Kazuhiro Takamatsu, Kazutoshi Nishiyama, Kyohei Fujita, Teppei Kamimura, Daisuke Ando, Toshihiro Ide, Takeshi Yoshimoto, Masayuki Shiozawa, Soichiro Matsubara, Yoshitaka Yamaguchi, Naoto Kinoshita, Takayuki Matsuki, Junji Takasugi, Keisuke Tokunaga, Kyoko Higashida, Kazunari Homma, Kazuomi Kario, Shoji Arihiro, Kazunori Toyoda for the SAMURAI Study Investigators

283. Anticoagulants, Reperfusion Therapy, and Outcomes in Ischemic Stroke Patients With Non-Valvular Atrial Fibrillation - A Single-Center, 6-Year Experience of 546 Consecutive Patients -

Satoshi Suda, Yuki Sakamoto, Seiji Okubo, Junya Aoki, Takashi Shimoyama, Takuya Kanamaru, Kentaro Suzuki, Akihito Kutsuna, Noriko Matsumoto, Chikako Nito, Yasuhiro Nishiyama, Masahiro Mishina, Kazumi Kimura

284. Anti-Xa Activity and Event Risk in Patients With Direct Factor Xa Inhibitors Initiated Early After Stroke Shinichi Wada, Kazunori Toyoda, Shoichiro Sato, Takayuki Matsuki, Takuya Okata, Masaya Kumamoto, Naoki Tagawa, Manabu Inoue, Akira Okamoto, Masafumi Ihara, Takanari Kitazono, Toshiyuki Miyata, Masatoshi Koga

Valvular Heart Disease (21)

285. Novel Risk Biomarker for Infective Endocarditis Patients With Normal Left Ventricular Ejection Fraction - Monocyte to High-Density Lipoprotein Cholesterol Ratio -

Xue-biao Wei, Feng Chen, Jie-leng Huang, Peng-cheng He, Yan-xing Wei, Ning Tan, Ji-yan Chen, Dan-qing Yu, Yuan-hui Liu

286. Transcatheter Aortic Valve Implantation Improves Cardiac Sympathetic Nerve Activity on ${ }^{123}$ I-Metaiodobenzylguanidine Myocardial Scintigraphy in Severe Aortic Valve Stenosis

Mitsuo Sobajima, Hiroshi Ueno, Hiroshi Onoda, Hiroyuki Kuwahara, Shuhei Tanaka, Ryuichi Ushijima, Nobuyuki Fukuda, Shigeki Yokoyama, Saori Nagura, Toshio Doi, Akio Yamashita, Kazuaki Fukahara, Hisakatsu Ito, Koichiro Kinugawa

287. Echocardiographic Assessment of Cardiac Structural and Functional Abnormalities in Patients With End-Stage Renal Disease Receiving Chronic Hemodialysis

Hiroshi Matsuo, Kaoru Dohi, Hirofumi Machida, Hideyuki Takeuchi, Toshikazu Aoki, Hiroyuki Nishimura, Masashi Yasutomi, Michiharu Senga, Takehiko Ichikawa, Kentaro Kakuta, Yasuhide Mizutani, Akiko Tanoue, Naoki Isaka, Kazuki Oosugi, Sukenari Koyabu, Masato Sakurai, Yoshihisa Fukui, Hitoshi Kakimoto, Tadafumi Sugimoto, Takahiro Ohnishi, Tomohiro Murata, Eiji Ishikawa, Ryuji Okamoto, Tomomi Yamada, Toru Ogura, Yuki Nishimura, Takashi Tanigawa, Shinsuke Nomura, Masakatsu Nishikawa, Masaaki Ito 
288. Acute Heart Failure in Patients With Severe Aortic Stenosis - Insights From the CURRENT AS Registry Kazuya Nagao, Tomohiko Taniguchi, Takeshi Morimoto, Hiroki Shiomi, Kenji Ando, Norio Kanamori, Koichiro Murata, Takeshi Kitai, Yuichi Kawase, Chisato Izumi, Makoto Miyake, Hirokazu Mitsuoka, Masashi Kato, Yutaka Hirano, Shintaro Matsuda, Tsukasa Inada, Tomoyuki Murakami, Yasuyo Takeuchi, Keiichiro Yamane, Mamoru Toyofuku, Mitsuru Ishii, Eri Minamino-Muta, Takao Kato, Moriaki Inoko, Tomoyuki Ikeda, Akihiro Komasa, Katsuhisa Ishii, Kozo Hotta, Nobuya Higashitani, Yoshihiro Kato, Yasutaka Inuzuka, Chiyo Maeda, Toshikazu Jinnai, Yuko Morikami, Naritatsu Saito, Kenji Minatoya, Takeshi Kimura on behalf of the CURRENT AS Registry Investigators

289. Definitive Determinant of Late Significant Tricuspid Regurgitation After Aortic Valve Replacement Shin Yajima, Daisuke Yoshioka, Koichi Toda, Satsuki Fukushima, Shigeru Miyagawa, Yasushi Yoshikawa, Shunsuke Saito, Keitaro Domae, Takayoshi Ueno, Toru Kuratani, Yoshiki Sawa

290. Transcatheter Aortic Valve Implantation (TAVI) for Native Aortic Valve Regurgitation - A Systematic Review Altayyeb Yousef, Zachary MacDonald, Trevor Simard, Juan J. Russo, Joshua Feder, Michael V. Froeschl, Alexander Dick, Christopher Glover, Ian G. Burwash, Azeem Latib, Josep Rodés-Cabau, Marino Labinaz, Benjamin Hibbert

291. Do Patients With High $\mathrm{CHA}_{2} \mathrm{DS}_{2}$-VASc Scores Need High Intensity of Anticoagulants After Valve Surgery? Hsi-Yu Yu, Ming-Hsien Lin, Lian-Yu Lin, Chih-Hsien Wang, Nai-Hsin Chi, Yih-Sharng Chen

292. Prevalence and Prognostic Significance of Functional Mitral and Tricuspid Regurgitation Despite Preserved Left Ventricular Ejection Fraction in Atrial Fibrillation Patients

Yukio Abe, Kanako Akamatsu, Kazato Ito, Yoshiki Matsumura, Kenji Shimeno, Takahiko Naruko, Yosuke Takahashi, Toshihiko Shibata, Minoru Yoshiyama

293. Minimally Invasive Mitral Valve Repair Through Right Minithoracotomy - 11-Year Single Institute Experience Taichi Sakaguchi, Toshinori Totsugawa, Masahiko Kuinose, Kentaro Tamura, Arudo Hiraoka, Genta Chikazawa, Hidenori Yoshitaka

Role of Computed Tomography in Planning the Appropriate X-Ray Gantry for Quantitative Aortography of Posttranscatheter Aortic Valve Implantation Regurgitation

Hiroki Tateishi, Yosuke Miyazaki, Takayuki Okamura, Rodrigo Modolo, Mohammad Abdelghani, Osama I.I. Soliman, Tetsuro Oda, Akihito Mikamo, Yoshinobu Onuma, Kimikazu Hamano, Masafumi Yano, Patrick W. Serruys

295. Learning Curve for Transcatheter Aortic Valve Implantation Under a Controlled Introduction System - Initial Analysis of a Japanese Nationwide Registry -

Nobuhiro Handa, Hiraku Kumamaru, Kei Torikai, Shun Kohsaka, Morimasa Takayama, Junjiro Kobayashi, Hisao Ogawa, Haruki Shirato, Kensuke Ishii, Kazuhisa Koike, Yoshimasa Yokoyama, Hiroaki Miyata, Noboru Motomura, Yoshiki Sawa on behalf of Japanese TAVR Registry Participants

296. Impact of Frailty Markers for Unplanned Hospital Readmission Following Transcatheter Aortic Valve Implantation Mike Saji, Ryosuke Higuchi, Tetsuya Tobaru, Nobuo Iguchi, Shuichiro Takanashi, Morimasa Takayama, Mitsuaki Isobe

297. Independent Effect of Low Flow on Outcomes in Patients Undergoing Aortic Valve Replacement for Severe Aortic Stenosis

Suguru Miyazaki, Kenji Kuwaki, Kan Kajimoto, Satoshi Matsushita, Shizuyuki Dohi, Taira Yamamoto, Hiroaki Hata, Atsushi Amano

298. Long-Term Results of Tricuspid Annuloplasty Using MC3 Ring for Functional Tricuspid Regurgitation Jae Woong Choi, Kyung Hwan Kim, Sue Hyun Kim, Sang Yoon Yeom, Ho Young Hwang, Ki-Bong Kim

299. Outcomes of Self-Expanding vs. Balloon-Expandable Transcatheter Heart Valves for the Treatment of Degenerated Aortic Surgical Bioprostheses - A Propensity Score-Matched Comparison -

Tomoki Ochiai, Sung-Han Yoon, Rahul Sharma, Masaki Miyasaka, Takahiro Nomura, Tanya Rami, Yoshio Maeno, Tarun Chakravarty, Mamoo Nakamura, Wen Cheng, Raj Makkar

300. Early Surgery vs. Surgery After Watchful Waiting for Asymptomatic Severe Aortic Stenosis Makoto Miyake, Chisato Izumi, Tomohiko Taniguchi, Takeshi Morimoto, Masashi Amano, Shunsuke Nishimura, Takeshi Kitai, Takao Kato, Kazushige Kadota, Kenji Ando, Yutaka Furukawa, Tsukasa Inada, Moriaki Inoko, Katsuhisa Ishii, Genichi Sakaguchi, Fumio Yamazaki, Tadaaki Koyama, Tatsuhiko Komiya, Kazuo Yamanaka, Noboru Nishiwaki, Naoki Kanemitsu, Toshihiko Saga, Tatsuya Ogawa, Shogo Nakayama, Hiroshi Tsuneyoshi, Atsushi Iwakura, Kotaro Shiraga, Michiya Hanyu, Nobuhisa Ohno, Atsushi Fukumoto, Tomoyuki Yamada, Junichiro Nishizawa, Jiro Esaki, Kenji Minatoya, Yoshihisa Nakagawa, Takeshi Kimura on behalf of the CURRENT AS Registry Investigators

301. Discongruence Index - Simple Indicator to Predict Prosthesis-Patient Mismatch After Transcatheter Aortic Valve Replacement -

Jose Alberto de Agustin, Fabian Islas, Pilar Jimenez-Quevedo, Luis Nombela-Franco, Andrea Rueda Liñares, Patricia Mahia, Pedro Marcos-Alberca, Eduardo Pozo, Jose Juan Gomez de Diego, Maria Luaces, Ivan-Javier Nuñez-Gil, Miguel Ángel Garcia-Fernandez, Antonio Fernandez-Ortiz, Carlos Macaya, Leopoldo Perez de Isla

302. Surgical as Opposed to Transcatheter Aortic Valve Replacement Improves Basal Interventricular Septal Hypertrophy Hidetoshi Yoshitani, Akihiro Isotani, Jae-Kwan Song, Shinichi Shirai, Hiromi Umeda, Jeong Yoon Jang, Takeshi Onoue, Misako Toki, Byung-Joo Sun, Dae-Hee Kim, Nobuyuki Kagiyama, Akihiro Hayashida, Jong-Min Song, Masataka Eto, Yosuke Nishimura, Kenji Ando, Michiya Hanyu, Kiyoshi Yoshida, Robert A. Levine, Yutaka Otsuji 
303. Effect of the Initial Strategy for Active Endocarditis Complicated With Acute Heart Failure

Ryohei Matsuura, Daisuke Yoshioka, Koichi Toda, Junya Yokoyama, Shigeru Miyagawa, Yasushi Yoshikawa, Taichi Sakaguchi, Hiroyuki Nishi, Toshihiro Funatsu, Masao Yoshitatsu, Osamu Monta, Hirotsugu Fukuda, Yoshiki Sawa for the Osaka Cardiovascular Research (OSCAR) Study Group

304. Prognostic Impact of Computed Tomography-Derived Abdominal Fat Area on Transcatheter Aortic Valve Implantation

Taishi Okuno, Keita Koseki, Toru Nakanishi, Kai Ninomiya, Daijiro Tomii, Tetsu Tanaka, Yu Sato, Akira Osanai, Kei Sato, Hideki Koike, Kazuyuki Yahagi, Satoru Kishi, Kota Komiyama, Jiro Aoki, Motoi Yokozuka, Sumio Miura, Kengo Tanabe

305. Transcatheter Aortic Valve Replacement for Severe Aortic Stenosis Complicated by Sigmoid Septum Kizuku Yamashita, Tomoyuki Fujita, Satsuki Fukushima, Yusuke Shimahara, Yuta Kume, Yorihiko Matsumoto, Naonori Kawamoto, Kimito Minami, Daijiro Kabata, Hideaki Kanzaki, Chisato Izumi, Toshihisa Anzai, Junjiro Kobayashi

Vascular Biology and Vascular Medicine (7)

306. Eicosapentaenoic Acid-Enriched High-Density Lipoproteins Exhibit Anti-Atherogenic Properties

Nobuaki Tanaka, Yasuhiro Irino, Masakazu Shinohara, Shigeyasu Tsuda, Takeshige Mori, Manabu Nagao, Toshihiko Oshita, Kenta Mori, Tetsuya Hara, Ryuji Toh, Tatsuro Ishida, Ken-ichi Hirata

307. $\mathrm{Ca}^{2+} /$ Calmodulin-Dependent Protein Kinase II $\gamma$-Dependent Serine727 Phosphorylation Is Required for TMEM16A $\mathrm{Ca}^{2+}$-Activated $\mathrm{Cl}^{-}$Channel Regulation in Cerebrovascular Cells

Cai-Xia Lin, Xiao-Fei Lv, Feng Yuan, Xiang-Yu Li, Ming-Ming Ma, Can-Zhao Liu, Jia-Guo Zhou, Guan-Lei Wang, Yong-Yuan Guan

308. Segmental Difference in Vasoreactivity of the Human Right Gastroepiploic Artery

Takeshi Kinoshita, Masashi Tawa, Tomoaki Suzuki, Tohru Asai, Tomio Okamura

309. Neuroprotection Mediated by Upregulation of Endothelial Nitric Oxide Synthase in Rho-Associated, Coiled-CoilContaining Kinase 2 Deficient Mice

Yukio Hiroi, Kensuke Noma, Hyung-Hwan Kim, Nikola Sladojevic, Corey E. Tabit, Yuxin Li, Guray Soydan, Salvatore Salomone, Michael A. Moskowitz, James K. Liao

310. Heparin Induces the Mobilization of Heart-Derived Multipotent Mesoangioblasts During Cardiac Surgery With Cardiopulmonary Bypass or Cardiac Catheterization

Yoshihiro Hata, Masayoshi Iwasaki, Keisuke Fujitaka, Haengnam Park, Daisuke Sato, Chiharu Enoki, Naoki Minato, Keita Horitani, Miyuki Nakano, Hiroshi Kishimoto, Kensaku Wada, Masamichi Koyanagi, Yasushi Adachi, Yoshihiro Yamamoto, Andreas M. Zeiher, Stefanie Dimmeler, Ichiro Shiojima

311. Assessment of Congenital Vascular and Organ Anomalies in Subjects With Thalidomide Embryopathy Using NonContrast Magnetic Resonance Angiography

Julius Matthias Weinrich, Rudolf Beyer, Lennart Well, Enver Tahir, Maria Lindemann, Undine Wilke, Gerhard Adam, Peter Bannas, Gunnar K. Lund

312. Akt1-Mediated Muscle Growth Promotes Blood Flow Recovery After Hindlimb Ischemia by Enhancing Heme Oxygenase-1 in Neighboring Cells

Yoshiro Onoue, Yasuhiro Izumiya, Shinsuke Hanatani, Toshifumi Ishida, Yuichiro Arima, Satoru Yamamura, Yuichi Kimura, Satoshi Araki, Masanobu Ishii, Taishi Nakamura, Yu Oimatsu, Kenji Sakamoto, Eiichiro Yamamoto, Sunao Kojima, Koichi Kaikita, Kenichi Tsujita

Rapid Communications

1. Transcatheter Aortic Valve Implantation for Degenerated 19-mm Aortic Bioprosthetic Valve

Kizuku Yamashita, Tomoyuki Fujita, Satsuki Fukushima, Yusuke Shimahara, Yuta Kume, Yorihiko Matsumoto, Naonori Kawamoto, Tatsuro Hitsumoto, Nobuyasu Ito, Yasuhiro Hamatani, Atsushi Okada, Hiroyuki Takahama, Makoto Amaki, Takuya Hasegawa, Yasuo Sugano, Hideaki Kanzaki, Toshihisa Anzai, Satoshi Yasuda, Junjiro Kobayashi

2. Three-Year Follow-up After the Great East Japan Earthquake in the Incidence of Out-of-Hospital Cardiac Arrest With Cardiac Origin Junya Sado, Kosuke Kiyohara, Taku Iwami, Yuri Kitamura, Emiko Ando, Tetsuya Ohira, Tomotaka Sobue, Tetsuhisa Kitamura

3. Adiponectin in Chronic Thromboembolic Pulmonary Hypertension Sarasa Isobe, Masaharu Kataoka, Takashi Kawakami, Keiichi Fukuda

4. Effects and Safety of Sodium Glucose Cotransporter 2 Inhibitors in Diabetes Patients With Drug-Refractory Advanced Heart Failure

Yoshihiro Seo, Masayoshi Yamamoto, Tomoko Machino-Ohtsuka, Tomoko Ishizu, Kazutaka Aonuma

5. Physical Activity Does Not Reduce Aortic Valve Stenosis Incidence Philip Sarajlic, Alicja Wolk, Magnus Bäck, Susanna C. Larsson

6. MitraClip in Patients With Mitral Regurgitation and Left Ventricular Ejection Fraction $<30 \%$ - Potential Implications for the Treatment of Patients in Japan -

Hidehiro Kaneko, Mitsunobu Kitamura, Michael Neuss, Maki Okamoto, Tobias Schmidt, Hannes Alessandrini, Karl-Heinz Kuck, Issei Komuro, Christian Frerker, Christian Butter 
7. Giant Fold Gastritis Induced by Epoprostenol Infusion in Patients With Pulmonary Arterial Hypertension Yohei Miura, Masaharu Kataoka, Tomohiro Chiba, Takumi Inami, Hideaki Yoshino, Toru Satoh

8. Use of Pulmonary Inhalants Remains Remarkably High After Atrial Septal Defect Closure Martina Nassif, Odilia I. Woudstra, Joey M. Kuijpers, Peter J. Sterk, Reindert P. van Steenwijk, Aeilko H. Zwinderman, Berto J. Bouma, Barbara J. Mulder, Robbert J. de Winter

Images in Cardiovascular Medicine

1. Bilateral Arm Gangrene Associated With Heparin-Induced Thrombocytopenia After Extracorporeal Cardiopulmonary Resuscitation

Kansuke Ito, Toshiaki Isogai, Makiko Takada, Tetsushi Tatsumi, Kazuaki Enatsu, Hiroyuki Tanaka, Tamotsu Tejima

2. Slow-Flow Phenomenon After Stent Deployment in Lipid Rich Plaque Harboring Cholesterol Crystals

Satoshi Honda, Yu Kataoka, Yasuhide Asaumi, Yoichi Goto, Teruo Noguchi, Satoshi Yasuda

3. Migration of Large Thrombus From the Left Atrial Appendage During Transesophageal Echocardiography in a Stroke Patient

Kyu-Hwan Park, Jong-Ho Nam, Jang-Won Son

4. Successful Vascular Intervention Without Embolic Complications in Budd-Chiari Syndrome Eiji Taguchi, Junjiroh Koyama, Masataka Kajiwara, Masayuki Inoue, Yoko Horibata, Kazuhiro Nishigami, Koichi Nakao, Tomohiro Sakamoto

5. Inferior Vena Cava Thrombi Caused by Enlarged, Solitary Hepatic Cyst Eiji Taguchi, Nobuhiro Nakanishi, Koichi Nakao, Tomohiro Sakamoto

6. Hydrophilic Polymer Embolism in the Lung as an Adverse Event - Autopsy Case of Thromboembolisms in Multiple Organs Associated With Heparin-Induced Thrombocytopenia Satsuki Komoda, Hiroaki Ozawa, Takeshi Yuasa

7. Very Late Strut Overhang on Coronary Angioscopy After Bioresorbable Vascular Scaffold Implantation in a Clinically Stable Patient

Takashi Fujimura, Tomohiro Kawasaki, Takeshi Kimura

8. Angioscopic Findings of Acute Myocardial Infarction After Kawasaki Disease Toru Miyoshi, Fumiyasu Seike, Hideo Kawakami, Akira Oshita, Hiroshi Matsuoka

9. Pyogenic Aortitis - Role of 18F-FDG Positron Emission Tomography/Computed Tomography in Aortic Infection Imaging -

Alejandro Travieso-González, Isidre Vilacosta, David Vivas, Carmen Olmos, Javier Higueras Nafría, María Jesús Pérez Castejón, Aída Ortega Candil, Adela Pelayo Alarcón, Luis Ortega Medina

10. Clinical Utility of Fractional Flow Reserve on Computed Tomography in Left Main Trunk Disease Taichi Okonogi, Tomohiro Kawasaki

11. Protruding In-Stent Mass After Bioresorbable Polymer Sirolimus-Eluting Stent - Ex Vivo Intravascular Imaging and Histopathology -

Rika Kawakami, Takahiro Imanaka, Masaharu Ishihara, Takako Kihara, Kenichi Fujii, Seiichi Hirota, Hiroyuki Hao

12. Thalidomide for Hereditary Hemorrhagic Telangiectasia With Pulmonary Arterial Hypertension Tomohisa Nakamura, Takeshi Ogo, Nobuhiro Tahara, Shigefumi Fukui, Akihiro Tsuji, Jin Ueda, Yoshihiro Fukumoto, Norifumi Nakanishi, Hisao Ogawa, Satoshi Yasuda

13. Adult Case of Parachute-Like Asymmetrical Mitral Valve Complicated by Mitral Stenosis and Thrombus Formation Yujiro Kawai, Takahito Yokoyama, Gentaku Hama, Yasuyuki Toyoda, Miki Horigome, Yasutoshi Tsuda, Yoshikazu Yazaki, Takahiro Takemura

14. Radiation-Induced Cardiomyopathy Incidentally Detected on Oncology ${ }^{18}$ F-Fluorodeoxyglucose Positron Emission Tomography

Go Kawamura, Hideki Okayama, Naoto Kawaguchi, Saki Hosokawa, Tetsuya Kosaki, Tatsuya Shigematsu, Tatsunori Takahashi, Yoshitaka Kawada, Go Hiasa, Tadakatsu Yamada, Hiroshi Matsuoka, Yukio Kazatani

15. ${ }^{18}$ F-Fluorodeoxyglucose Positron Emission Tomography 10 Days Before Onset of Aortic Dissection Toshihiro Tsuruda, Shigeki Nagamachi, Masashi Yamaguchi, Sumiharu Sakamoto, Tetsunori Ishikawa, Kazuo Kitamura

16. Coexistence of a Double-Chambered Right Ventricle With Ebstein-Like Anomaly and Right Ventricular Apical Hypoplasia - Usefulness of Multimodality Imaging -

Kyohei Marume, Shigefumi Fukui, Keiko Shimamoto, Hideo Ohuchi, Akihiro Tsuji, Takeshi Ogo, Norifumi Nakanishi, Satoshi Yasuda

17. Huge Metastatic Gastrointestinal Stromal Tumor Occupying the Right Ventricle Yudai Tamura, Hayato Tada, Hiroki Kato, Kenji Iino, Kenshi Hayashi, Masa-aki Kawashiri, Hirofumi Takemura, Masakazu Yamagishi

18. Exercise Ischemia Induced by Essential Thrombocytemia Diagnosed on Treadmill Test - Transcutaneous Oxygen Pressure Measurement of Foot Ischemia Loukman Omarjee, Olivier Stivalet, Guillaume Mahe, Vincent Jaquinandi 
19. Severe Aortic and Mitral Stenosis Secondary to Slowly Progressive Hunter Syndrome in an Elderly Patient Yosuke Takahashi, Takashi Murakami, Hiromichi Fujii, Masanori Sakaguchi, Shinsuke Nishimura, Daisuke Yasumizu, Yoshito Sakon, Yuki Kubo, Masahiko Osawa, Toshihiko Shibata

20. Perforation of the Left Atrial Appendage Caused by Inadvertent Deployment of a Soft J-Tipped Guidewire During Radiofrequency Hot-Balloon Ablation

Hiro Yamasaki, Fumi Yamagami, Takeshi Machino, Kenji Kuroki, Yukio Sekiguchi, Kazutaka Aonuma, Akihiko Nogami

21. Ventricular Fibrillation Due to Early Repolarization Syndrome in the Wake of Hypothermia Due to Fulminant Acute Disseminated Encephalomyelitis

Kyoichiro Yazaki, Yoichi Ajiro, Kunihiko Shimizu, Fumiaki Mori, Nobue Yagihara, Akinori Sato, Tatsuya Takahashi, Kazunori Iwade

22. Subacute Left Atrial Thrombus Formation After Percutaneous Edge-to-Edge Mitral Valve Repair for Functional Mitral Regurgitation

Makoto Amaki, Yu Kataoka, Hideaki Kanzaki, Koji Miyamoto, Atsushi Okada, Yasuhiro Hamatani, Masashi Fujino, Hiroyuki Takahama, Takuya Hasegawa, Yasuo Sugano, Kengo Kusano, Yoshihiko Ohnishi, Tomoyuki Fujita, Junjiro Kobayashi, Toshihisa Anzai, Satoshi Yasuda

23. Extensive Calcification of the Radial and Ulnar Arteries Associated With Vasculitis Shoko Nakagawa, Osami Kawarada, Takeshi Yagyu, Teruo Noguchi, Satoshi Yasuda

24. Different Behavior of Bioresorbable Vascular Scaffold and Metallic Stent With Positive Vessel Remodeling Satoru Mitomo, Damiano Regazzoli, Luciano Candilio, Akihito Tanaka, Azeem Latib, Antonio Colombo

25. Mitral Valve Systolic Anterior Motion-Associated Sounds in Hypertrophic Cardiomyopathy Chieko Sakai, Tatsuya Kawasaki, Michiyo Yamano, Hirokazu Shiraishi, Tadaaki Kamitani, Satoaki Matoba

26. Bipolar Endo-Epicardial Radiofrequency Ablation of Arrhythmia Originating From the Left Ventricular Summit Piotr Futyma, Agnieszka Wysokińska, Jarosław Sander, Marian Futyma, Piotr Kułakowski

27. Intracoronary Acetylcholine Provocation Test via Coronary Bypass Graft Yuichi Saito, Yoshio Kobayashi

28. Progressive Mycotic Celiac Artery Aneurysm Associated With Coagulase-Negative Staphylococcal Prosthetic Valve Endocarditis

Makoto Tanaka, Takashi Kohno, Hideaki Obara, Seishi Nakatsuka, Takahiko Nishiyama, Nobuhiro Nishiyama, Hikaru Tsuruta, Mitsushige Murata, Yuichiro Maekawa, Akihiro Yoshitake, Motoaki Sano, Hideyuki Shimizu, Keiichi Fukuda

29. Life-Threatening Ventricular Arrhythmia Following Exercise-Induced Vasospastic Myocardial Ischemia at the Site of a Myocardial Bridge With Progressive J-Wave Manifestation Toru Adachi, Akira Sato, Akihiko Nogami, Kazutaka Aonuma

30. Utility of Single-Photon Emission Computed Tomography/Computed Tomography Fusion Imaging With ${ }^{99 \mathrm{~m} T c-}$ Pyrophosphate Scintigraphy in the Assessment of Cardiac Transthyretin Amyloidosis Noriko Tsuda, Shinya Shiraishi, Seitaro Oda, Koji Ogasawara, Fumi Sakamoto, Seiji Tomiguchi, Koichiro Fujisue, Seiji Takashio, Yasuhiro Izumiya, Kenichi Tsujita, Yukio Ando, Yasuyuki Yamashita

31. Identification of Undeployed Stent in the Left Main Coronary Artery After 3 Years on Optical Coherence Tomography

Thibault Ronchard, Nicolas Combaret, Guilhem Malcles, Pascal Motreff, Géraud Souteyrand

32. Cardiovascular Structural Interventions - Echo/Computed Tomography-Fluoroscopy Fusion Imaging Atlas Manuel Barreiro-Perez, Ignacio Cruz-Gonzalez, Jose Carlos Moreno-Samos, Monica Fuertes Barahona, Pedro L. Sanchez

33. Neoatherosclerosis With Napkin Ring Calcification Aritomo Katsura, Yoshiyasu Minami, Taiki Tojo, Junya Ako

34. Transcatheter Mitral Valve Prosthesis Dysfunction - Early Valve Degeneration or Thrombosis? Allan Böhm, Vasil Hricak, Branislav Tomasovic, Martin Bena, Peter Michalek, Jan Postulka

35. Percutaneous Pulmonary Valve Implantation Contraindicated by Severe Aortic Regurgitation Due to Left Coronary Sinus Deformation

Alessia Faccini, Luca Giugno, Mario Carminati, Gianfranco Butera

36. Rare Case of Giant Non-Thrombosed Coronary Artery Aneurysm at the Left Anterior Descending Coronary Artery Yutaka Matsuhiro, Koji Yasumoto, Keisuke Yasumura, Akihiro Tanaka, Naoki Mori, Daisuke Nakamura, Masamichi Yano, Yasuyuki Egami, Ryu Shutta, Jun Tanouchi, Masami Nishino

37. Localized Doxorubicin-Induced Cardiomyopathy Complicated With Shower Emboli Originating From Apical Intramural Thrombi

Hironori Ishiguchi, Shigeki Kobayashi, Shinichi Okuda, Takayuki Okamura, Munemasa Okada, Genzou Takemura, Masaya Takahashi, Akihito Mikamo, Kimikazu Hamano, Masafumi Yano

38. Vascular Digit Injury in a College Baseball Player

Tatsuya Maruhashi, Yasuki Kihara, Yukihito Higashi

39. Large Non-Bacterial Vegetation Causing Acute Aortic Regurgitation - Unexpected Finding at Autopsy Toshiki Sawai, Munenari Ikezawa, Atsuya Hirota, Shigetada Yamamoto, Hiroshi Nakajima, Katsutoshi Makino, Kojiro Takase, Masaaki Ito 
40. Improvement of Libman-Sacks Endocarditis With Combined Hydroxychloroquine-Vitamin K Antagonist Therapy in a Primary Antiphospholipid Syndrome Patient

Loukman Omarjee, Vincent Jaquinandi, Audrey Camarzana, Frederic Rouleau, Tin-Hinan Mezdad, Thierry Le Tourneau, Guillaume Mahe, Cristina Belizna

41. Aortic Dissection Resulting in Simultaneous Bilateral Coronary Invasion via Different Mechanisms on Intravascular Ultrasonography Atsushi Sugiura, Hideki Kitahara, Kenichi Fujii, Yoshihide Fujimoto, Yoshio Kobayashi

42. Yellow Nail Syndrome Complicating Coronary Artery Bypass Graft Surgery Taku Omori, Ryuji Okamoto, Hajime Fujimoto, Jun Masuda, Tetsu Kobayashi, Eitaro Fujii, Yoshiyuki Takei, Masaaki Ito

43. Vascular Network Inside the Heart - Collateral Flow on Color Doppler Echo in a Child With Anomalous Left Coronary Artery From the Pulmonary Artery (ALCAPA) -

Lisheng Lin, Takashi Murakami, Junko Shiono, Hitoshi Horigome

44. Rapid-Growing Right Atrial Myxoma 7 Months After Catheter Ablation Under Anticoagulation Therapy - Serial Echocardiography and Computed Tomography -

Nobuhiko Haruki, Takeshi Onohara, Daiki Tsujimoto, Kazuhiko Iitsuka, Yoshiharu Kinugasa, Masahiko Kato, Motonobu Nishimura, Kazuhiro Yamamoto

45. Overwhelming Suppression of Neointimal Coverage on High-Resolution Coronary Angioscopy After PaclitaxelCoated Balloon Angioplasty for In-Stent Restenosis of Cobalt-Chrome Everolimus-Eluting Stent Yuhei Nojima, Shinsuke Nanto, Hidenori Adachi, Madoka Ihara, Tetsuya Kurimoto

46. Cannon Sounds Kuniyasu Harimoto, Tatsuya Kawasaki, Tadaaki Kamitani

47. Pathological Findings of Rivaroxaban-Associated Hemorrhagic Pericarditis Masaki Kodaira, Hiroyuki Yamazaki, Ryota Tabei, Toshiki Kuno, Kazuhiko Shimizu, Yohei Numasawa

48. Primary Cardiac Diffuse Large B-Cell Lymphoma Shu Yoshihara, Masaki Matsunaga, Fumihiko Tanioka, Masaaki Naito

49. Diastolic Tricuspid Regurgitation Related to Atrial Systole With a Bileaflet Mechanical Valve Akihito Saito, Katsura Soma, Toshiya Kojima, Toshiro Inaba, Atsushi Yao, Issei Komuro

50. Impella 5.0 as a Bridge to Implantable Left Ventricular Assist Device - First Clinical Case in Japan Shohei Yoshida, Koichi Toda, Shigeru Miyagawa, Yasushi Yoshikawa, Hiroki Hata, Kei Torikai, Kazuo Shimamura, Shunsuke Saito, Keiwa Kin, Koichi Maeda, Keitaro Domae, Yoshiki Watanabe, Ryohei Matsuura, Kenta Masada, Shungo Hikoso, Isamu Mizote, Fusako Sera, Kei Nakamoto, Taito Masawa, Yasushi Sakata, Toru Kuratani, Yoshiki Sawa

51. Bidirectional Shunt Trajectory in Ventricular Septal Defect With Eisenmenger's Syndrome

Hiromi Kayamori, Takeshi Kashimura, Yosuke Horii, Tsutomu Kanazawa, Toshio Fujita, Naoki Kubota, Yasuhiro Ikami, Toshiki Takano, Takeshi Okubo, Makoto Hoyano, Takao Yanagawa, Takuya Ozawa, Kazuyuki Ozaki, Tohru Minamino

52. Enzyme Replacement Therapy Provides Effective, Long-Term Treatment of Cardiomyopathy in Pompe Disease Shiro Baba, Daisuke Yoshinaga, Kentaro Akagi, Koichi Matsuda, Atsushi Yokoyama, Takeshi Yoshida, Takuya Hirata

53. Cardiac Amyloidosis Mimicking Dilated Cardiomyopathy But Showing Relative Apical Sparing of Longitudinal Strain

Miwa Ishida, Yoko Yamada, Tomohiro Mizutani, Atsuko Hara, Yoshiki Sekijima, Junya Ako, Takayuki Inomata

54. Recurrent Hypotension During Hemodialysis Associated With Double Chamber Right Ventricle in Hypertrophic Cardiomyopathy

Won-Jong Park, Jang-Won Son, Chan-Hee Lee, Jung-Hee Lee, Ung Kim, Jong-Seon Park, Dong-Gu Shin

55. Utility of Coronary Computed Tomography Angiography in the Diagnosis and Management of Acute-Phase AdultOnset Kawasaki Disease

Toshiki Kuno, Akimichi Shibata, Masaki Kodaira, Yohei Numasawa

56. Leadless Pacemaker and Subcutaneous Implantable Cardioverter Defibrillator Combination in a Hemodialysis Patient

Yoshinari Enomoto, Hikari Hashimoto, Rina Ishii, Shunsuke Torii, Keijiro Nakamura, Mahito Noro, Kaoru Sugi, Masao Moroi, Masato Nakamura

\section{JCS Guidelines}

1. Guidelines for Heart Disease Screening in Schools (JCS 2016/JSPCCS 2016) - Digest Version -

Naokata Sumitomo, Reizo Baba, Shozaburo Doi, Takashi Higaki, Hitoshi Horigome, Fukiko Ichida, Hiromi Ishikawa, Mari Iwamoto, Naomi Izumida, Yuji Kasamaki, Keisuke Kuga, Yoshihide Mitani, Haruki Musha, Toshio Nakanishi, Masao Yoshinaga, Katsumi Abe, Mamoru Ayusawa, Tatsunori Hokosaki, Taichi Kato, Yoshiaki Kato, Kunio Ohta, Hirofumi Sawada, Hiroya Ushinohama, Shigeki Yoshiba, Hirotsugu Atarashi, Atsushi Hirayama, Minoru Horie, Masami Nagashima, Koichiro Niwa, Shunichi Ogawa, Ken Okumura, Hiroyuki Tsutsui on behalf of the Japanese Circulation Society and the Japanese Society of Pediatric Cardiology and Cardiac Surgery of Joint Working Group 
1. Where Are the Secrets of Increased Thrombosis and Aneurysm Formation With the Current Bioresorbable Vascular Scaffolds Hidden?

(Letter) Nicholas G Kounis, Ioanna Koniari, Grigorios Tsigkas, George D Soufras, George Hahalis

(Reply) Jeehoon Kang, Hyo-Soo Kim

…... 608

…... 609

2. Identification of Pathogenic Mutations for Dilated Cardiomyopathy Accompanied With Unicuspid Aortic Valve (Letter) Hiroyuki Morita, Issei Komuro (Reply) Haruhiko Higashi, Akiyoshi Ogimoto

........ 1723

….... 1724

3. Is There Any Clinical Significance of the Acute Antral Lesion Size After Pulmonary Vein Isolation Using Different Balloon Technologies or Ablation Energy?

(Letter) Tolga Aksu, Tümer Erdem Guler, Serdar Bozyel, Kazım Serhan Ozcan, Kivanc Yalin

4. Serum 1,25-Dihydroxyvitamin D Level Is Inappropriate for Use in Prospective Studies of Cancer Incidence (Letter) William B. Grant

(Reply) Kaoru Umehara, Naoko Mukai, Takanari Kitazono, Sumio Hoka, Yutaka Kiyohara, Toshiharu Ninomiya

5. Knocking the Chest as a "Bridge to Pacemaker": Treatment of Bradyasystole by Percussion Pacing

(Letter) Hendrik Drinhaus, Andreas Hohn, Thorsten Annecke

(Reply) Takeshi Wada, Mihoko Hagiwara-Nagasawa, Katsunori Yoshihara, Yuji Nakazato, Keith G. Lurie, Atsushi Sugiyama

6. Coronary Computed Tomography Angiography-Derived Fractional Flow Reserve Assessment: Many Roads to Reach the Same Goal

(Letter) Stefan Baumann, Dirk Lossnitzer, Matthias Renker, Martin Borggrefe, Ibrahim Akin

(Reply) Hiromasa Otake, Charles A. Taylor, Hitoshi Matsuo, Nobuhiro Tanaka, Takashi Akasaka

7. Association Between Aplastic Anemia and Atrial Fibrillation: Is Inflammation the Only Underlying Mechanism? (Letter) Yusuf Ziya Şener, Metin Okşul, Cem Çöteli (Reply) Wei-Syun Hu, Fung-Chang Sung, Cheng-Li Lin

….... 2216

….... 2445

…... 2446

….... 2448

….... 2449

…... 2450

….... 2451

8. Myocardial Deformation Analysis and Late-Gadolinium Enhancement: Important Markers of Cardiac Amyloidosis Involvement That Can Masquerade as a False-Negative Diagnosis

(Letter) Gianluca Di Bella, Fausto Pizzino

(Reply) Seitaro Oda

9. Imbalanced Angiogenesis in Peripartum Cardiomyopathy (PPCM) (Letter) Julie A Damp, Zolt Arany, James D Fett, Lori Blauwet, Uri Elkayam

10. Ketone Bodies Are an Alternative Energy Source and Exert Antioxidant Effects (Letter) Yoshiyuki Hattori

11. Bioresorbable Vascular Scaffolds: Is the Light Fading at the End of the Tunnel? (Letter) Ahmed N. Mahmoud, Akram Y. Elgendy, Islam Y. Elgendy (Reply) Jeehoon Kang, Hyo-Soo Kim

12. MitraClip in Patients With Severe Mitral Regurgitation and Severe Left Ventricular Systolic Dysfunction (Letter) Ching-Wei Lee, Shih-Hsien Sung, Wei-Ming Huang, Chiao-Po Hsu, Kuo-Piao Chung

13. Harmonization by Doing Proposal for Global Clinical Trial Designs for Endovascular Devices for Treatment of Critical Limb Ischemia: The United States Food and Drug Administration Perspective

(Letter) Kenneth J. Cavanaugh Jr, Donna C. Buckley, Misti L. Malone

(Reply) Hiroyoshi Yokoi, Mami Ho, Shin Iwamoto (on behalf of the authors)

\section{Corrigendum}

1. Electrophysiological Characteristics and Radiofrequency Catheter Ablation Treatment of Idiopathic Ventricular Arrhythmias Successfully Ablated From the Ostium of the Coronary Sinus Yoshiaki Yui, Yukio Sekiguchi, Akihiko Nogami, Hiro Yamasaki, Takeshi Machino, Kenji Kuroki, Miyako Igarashi, Kazutaka Aonuma

2. Amniotic Fluid Natriuretic Peptide Levels in Fetuses With Congenital Heart Defects or Arrhythmias Takekazu Miyoshi, Hiroshi Hosoda, Takashi Umekawa, Takashi Asada, Akihiro Fujiwara, Ken-ichi Kurosaki, Isao Shiraishi, Michikazu Nakai, Kunihiro Nishimura, Mikiya Miyazato, Kenji Kangawa, Tomoaki Ikeda, Jun Yoshimatsu, Naoto Minamino 